VOL. 60 (1999) [429-458]

\title{
SLICE CONVERGENCE OF PARAMETRISED SUMS OF CONVEX FUNCTIONS IN NON-REFLEXIVE SPACES
}

\author{
Robert Wenczel AND ANDREW Eberhard
}

\begin{abstract}
The objectives of this study of slice convergence are two-fold. The first is to derive results regarding the passage of certain semi-convergences through Young-Fenchel conjugation. These semi-convergences arise from the splitting of the usual slice topology in the primal and dual spaces into (non-Hausdorff) topologies: the upper slice topology $\tau_{s}^{+}$; a topology $\tau_{*}^{+}$generating a convergence closely resembling the bounded-weak ${ }^{*}$ upper Kuratowski convergence; along with the respective primal and dual lower Kuratowski topologies. This gives rise to topological convergences not reliant on sequentially-based definitions found in many such studies, and associated topological continuity results for conjugation (in normed spaces), in contrast to the usual sequential continuity exhibited by analogues of Mosco convergence. The second objective is to study the passage of slice convergence through addition. Such sum theorems have been derived in other works and we establish previous theorems from a unified framework as well as obtaining a new result.
\end{abstract}

\section{INTRODUCTION}

Within the context of reflexive Banach spaces Mosco-convergence has shown itself to be the natural variational convergence to use in the study of approximation and convergence of infima of convex functionals [1]. This is largely due to the fact that the Young-Fenchel conjugation $f \mapsto f^{*}$ defined on $\Gamma(X)$, the proper lower semi-continuous convex functions, is bi-continuous with respect to the associated Mosco topology. However, in [8] it is shown that even sequential continuity must fail when the underlying space is not reflexive. In the context of non-reflexive spaces slice convergence displays many of the desirable properties that Mosco convergence does in reflexive spaces [6]. Another variational convergence which has attracted attention in the non-reflexive context is that induced by the Attouch-Wets or epi-distance topology [7]. Despite the applicability of this metrisable topology to the quantitative analysis of stability and convergence of infima it has one undesirable property from the point of view of approximation. In order for a increasing sequence (in the sense of set inclusion) of finite-dimensional subsets

Received 22nd February, 1999

Research of the authors was supported by an ARC large grant.

Copyright Clearance Centre, Inc. Serial-fee code: 0004-9727/99 \$A2.00+0.00. 
$\left\{C_{n}\right\}_{n \in \mathbf{N}}$ to epi-distance converge to another set $C$ then the limit set must be locally compact with respect to the strong topology. On the other hand slice convergence of such a sequence only implies separability of $C$. Unfortunately this gain comes at a cost. One requires more stringent assumptions to deduce slice convergence of a sum $\left\{f_{v}+g_{v}\right\}_{v \in W}$ of proper convex functions $f_{v}, g_{v} \in \Gamma(X)$ from the slice convergence of the individual families $\left\{f_{v}\right\}_{v \in W}$ and $\left\{g_{v}\right\}_{v \in W}$ (here $W$ is some topological space containing $w$ and $v \rightarrow w$ ). Such results will be referred to as "sum theorems". Some authors have preferred to study the problem of convergence of $\left\{N_{v} \cap M_{v}\right\}_{v \in W}$, the intersection of two convergent families of closed convex sets $\left\{N_{v}\right\}_{v \in W}$ and $\left\{M_{v}\right\}_{v \in W},[5,19]$. Such results can easily be deduced from a sum theorem by simply choosing each of $f_{v}$ and $g_{v}$ to be the indicators of the closed convex sets $N_{v}$ and $M_{v}$, respectively. Indeed it is possible to deduce such sum theorems from results regarding the convergence of intersection of sets. Thus either point of view has equal utility. Sum theorems are important as they enable one to study the approximation and convergence of abstract convex optimisation problems [2, 9]. Such results are readily applicable to specific problems as we demonstrate in our example in Section 5 .

In this paper we restrict our attention to the study of sum theorems for slice convergence. Indeed much weaker assumptions may be made to ensure such a sum theorem holds for epi-distance convergence (see [5] and [7, Section 7.4 and the associated exercise set 8.1]). One could argue that sum theorems for epi-distance convergence have been more extensively studied than those for slice convergence, an observation which motivates this study. Our main tools are the bi-continuity of Young-Fenchel conjugation with respect to the slice topology and the properties of the infimal convolution

$$
(f \square g)(x):=\inf _{y \in X}(f(y)+g(x-y))
$$

with respect to conjugation. The slice topology $\tau_{s}$ may be viewed (see [7]) as the join of two non-Hausdorff topologies, one of which is the topology corresponding to the lower Kuratowski convergence of epigraphs. The other half of slice convergence is associated with a topology we shall refer to as the "upper slice topology" (denoted by $\tau_{s}^{+}$). This is a topology on the hyperspace $C(X \times \mathbf{R})$ of all (nonempty) closed convex subsets of $X \times \mathbf{R}$, generated by the subbasis consisting of all sets of the form

$$
\left(B^{c}\right)^{++}:=\left\{A \mid A \text { closed convex with } A+B(0, \varepsilon) \subseteq B^{c} \text { for some } \varepsilon>0\right\},
$$

where $B$ is a closed bounded and non-empty convex subset of $X \times \mathbf{R}$ and $B^{c}$ denotes its complement. Then we say $f \in \tau_{s}^{+}-\lim _{v \rightarrow w} f_{v}$ when:

$$
\text { epi } f_{v} \in\left(B^{c}\right)^{++} \text {for all } v \text { near } w \text { whenever epi } f \in\left(B^{c}\right)^{++} \text {. }
$$

The join of these two topologies gives rise to the (Hausdorff) topology inducing slice convergence and it is usually this convergence which is studied. Similar considerations 
apply to the dual slice topology $\tau_{s}^{*}$. The two associated non-Hausdorff topologies are often better viewed as inducing semi-continuities rather than convergences and this is the point of view we take here. In the development of our sum theorems we shall need to understand how the Young-Fenchel conjugation interacts with such (topological) semi-continuities. This is investigated in Section 3. The results presented here yield (in the context of normed spaces) topological versions of existing sequential continuity results $[1,4,24]$, without reflexivity or even completeness of the underlying space. (The cited results were derived with a view to proving sequential bi-continuity for Young-Fenchel conjugation relative to Mosco convergence). In the context of the slice topology, this appears to be the first time such a study has been undertaken (for the component topologies of $\tau_{s}$ ) in that it is usually only the interaction of conjugation with the join of these topologies (that is, the slice topology $\tau_{s}$ ) which is considered. From this point of view the results presented here also augment those already found in the literature on slice convergence $[6,7]$.

In Section 4 we apply this machinery to the development of sum theorems for slice convergence. Our goal here is to deduce a number of results already appearing in the literature from one unified approach for the case of Banach spaces. Indeed we are able to deduce results previously obtained by Lahrache [7, page 275] and Penot [19] in Banach spaces. In doing so we are able to obtain a new result using the notion of compactly epi-Lipschitz sets, see $[10,17,18]$. Denote by $\operatorname{rec} C$ the cone of recession directions of the convex set $C$. We say a family $\left\{g_{v}\right\}_{v \in W}$ has uniformly compactly epi-Lipschitz recession cones if there exists a compact set $H$ in $X, r>0$ and neighbourhood $W$ of $w$ such that for all $v \in W$ we have

$$
B(0, r) \subseteq \operatorname{rec} g_{v}-H
$$

for all $v \in W$ where $\operatorname{rec} g_{v}:=\operatorname{rec}\left\{x \in X \mid g_{v}(x) \leqslant \alpha\right\}$. Note that for $g_{v} \in \Gamma(X)$ the cone rec $g_{v}$ is independent of $\alpha$. If we also assume cone $\left(\operatorname{dom} f_{w}-\operatorname{dom} g_{w}\right)=X$ and that the following weak* and strong lower closures coincide;

$$
\overline{f_{w}^{*} \square g_{w}^{*}} \omega^{*}=\overline{f_{w}^{*} \square g_{w}^{*}}
$$

then slice convergence of $\left\{f_{v}\right\}_{v \in W}$ and $\left\{g_{v}\right\}_{v \in W}$ implies that of $\left\{f_{v}+g_{v}\right\}_{v \in W}$. The lower closure condition (1.2) is satisfied immediately if $X$ is reflexive, and is also implied by a number of conditions including the strengthened interiority condition $0 \in \operatorname{core}\left(\operatorname{dom} f_{w}\right.$ $\operatorname{dom} g_{w}$ ) (that is, cone $\left(\operatorname{dom} f_{w}-\operatorname{dom} g_{w}\right)=X$ ) or when both $f_{v}$ and $g_{v}$ are uniformly bounded below. When $g_{v}$ is the indicator of a linear subspace $N_{v} \subseteq X$ the condition (1.1) corresponds to the following uniform finite-codimensionality assumption: There exists a finite-dimensional subspace $F \subseteq X^{*}$ and a neighbourhood $V$ of $w$ such that $\bigcup_{v \in V} N_{v}^{\perp} \subseteq F$. From [7, Exercise 8.1.6] we know that slice convergence of the intersection of two families of slice-converging subspaces $\left\{M_{v}\right\}_{v \in V}$ and $\left\{N_{v}\right\}_{v \in V}$ may fail when neither of these spaces 
satisfy this uniform finite-codimensionality condition. Thus the condition (1.1) cannot be omitted in general from this sum theorem.

\section{Preliminaries}

In this section we draw together a number of results and definitions. This is done to make the development self-contained. A reader conversant with set-convergence notions and the infimal convolution need only read the first part of this section, only returning to consult results and definitions as needed.

We shall let $C(X)$ stand for the class of all nonempty closed convex subsets of a normed space $X$ and $C B(X)$ the closed bounded convex sets. Place $d(a, B)=$ $\inf \{\|a-b\| \mid b \in B\}, B(0, \rho)=\{x \in X \mid\|x\|<\rho\}$ and $\bar{B}(0, \rho)=\{x \in X \mid\|x\| \leqslant \rho\}$. Corresponding balls in the dual space $X^{*}$ will be denoted $B^{*}(0, \rho)$ and $\bar{B}^{*}(0, \rho)$ respectively. The indicator function of a set $A$ will be denoted $\delta_{A}$, and $S(A, \cdot)$ will denote the support function. The polar of $A \subseteq X$ will be written as $A^{\circ}$, and for $A \subseteq X^{*},{ }^{\circ} A$ denotes the polar as a subset of $X$. We shall use u.s.c. to denote upper-semicontinuity and l.s.c. to denote lower-semicontinuity. Recall that a function $f: X \rightarrow \overline{\mathbf{R}}$ is called closed, proper convex on $X$ if and only if $f$ is convex, l.s.c., is never $-\infty$, and is not identically $+\infty$. The class of all closed proper convex functions on $X$ is denoted by $\Gamma(X)$, and $\Gamma^{*}\left(X^{*}\right)$ denotes the class of all weak ${ }^{*}$ closed proper convex functions on $X^{*}$. We shall use the notation $\bar{A}$ for the closure of a set $A$ in a topological space $(Z, \tau)$ and, to emphasise the topology, we may write $\bar{A}^{r}$. For $x \in Z, \mathcal{N}_{\tau}(x)$ denotes the collection of all $\tau$-neighbourhoods of $x$. For a function $f: Z \rightarrow \overline{\mathbf{R}}$, the epigraph of $f$, denoted epi $f$, is the set $\{(x, \alpha) \in Z \times \mathbf{R} \mid f(x) \leqslant \alpha\}$, and the strict epigraph epi $_{s} f$ is the set $\{(x, \alpha) \in Z \times \mathbf{R} \mid f(x)<\alpha\}$. The domain, denoted dom $f$ is the set $\{x \in Z \mid f(x)<+\infty\}$. The (sub-)level set $\{x \in Z \mid f(x) \leqslant \alpha\}$ (where $\alpha>\inf _{Z} f$ ) will be given the abbreviation $\{f \leqslant \alpha\}$. Any product $X \times Y$ of normed spaces will always be understood to be endowed with the box norm $\|(x, y)\|=\max \{\|x\|,\|y\|\}$; any balls in such product spaces will always be with respect to the box norm. We also shall assume the following convention for products $Z \times \mathbf{R}$ where $(Z, \tau)$ is topological: We assume the product topology, where $\mathbf{R}$ has the usual topology, and for any subset $C \subseteq Z \times \mathbf{R}$, its closure in this topology is written as $\bar{C}^{\tau}$. If $f:(Z, \tau) \rightarrow \overline{\mathbf{R}}$, its $\tau$-l.s.c. hull, denoted $\bar{f}^{\tau}$, is defined by $\bar{f}^{\tau}(x)=\liminf _{x^{\prime} \rightarrow x} f\left(x^{\prime}\right)$. The (extended) lower closure $\underline{\mathrm{cl}}_{\tau} f$ is defined to coincide with $\bar{f}^{r}$ if the latter does not take the value $-\infty$ anywhere, and to be identically $-\infty$ otherwise.

Definition 2.1: Let $F: W \rightarrow 2^{X}$ be a multifunction from topological spaces $W$ to $X$.

$$
\text { 1. } \limsup _{v \rightarrow w} F(v)=\bigcap_{V \in \mathcal{N}(w)} \overline{\bigcup_{v \in V} F(v)}
$$


2. $\liminf _{v \rightarrow w} F(v)=\bigcap_{\{B \subseteq W \mid w \in \bar{B}\}} \overline{\bigcup_{v \in B} F(v)}$

3. $\quad F(\cdot)$ is lower-semicontinuous at $w$ if and only if $F(w) \subseteq \liminf _{v \rightarrow w} F(v)$. For a net $\left\{A_{\lambda}\right\}_{\lambda \in I}$ of subsets of $X$

4. $\quad \limsup _{\lambda} A_{\lambda}=\bigcap_{J \text { residual in } I} \overline{\bigcup_{\lambda \in J} A_{\lambda}}$;

5. $\liminf _{\lambda} A_{\lambda}=\bigcap_{J \text { cofinal in } I} \overline{\bigcup_{\lambda \in J} A_{\lambda}}$.

REMARK 2.1. It is easily seen that this notion of lower-semicontinuity is equivalent to the classical formulation-namely: For any open set $U$ intersecting $F(w)$ there is a neighbourhood $V$ of $w$ for which $F(v) \cap U$ is nonempty for every $v$ in $V$.

REMARK 2.2. For metrisable $X$, the above definitions can be shown to have the equivalent forms:

1. $\limsup _{v \rightarrow w} F(v)=\left\{x \in X \mid \exists\right.$ a net $v_{\beta} \rightarrow w$ and $x_{\beta} \in F\left(v_{\beta}\right)$ with $\left.x_{\beta} \rightarrow x\right\}$ $=\left\{x \in X \mid \liminf _{v \rightarrow w} d(x, F(v))=0\right\}$.

2. $\liminf _{v \rightarrow w} F(v)=\left\{x \in X \mid \forall\right.$ nets $v_{\beta} \rightarrow w, \exists x_{\beta} \rightarrow x$ with $x_{\beta} \in F\left(v_{\beta}\right)$ $=\left\{x \in X \mid \limsup _{v \rightarrow w} d(x, F(v))=0\right\}$ eventually $\}$

with obvious analogs for nets of sets.

Definition 2.2: Let $A$ be a convex set in a topological vector space and $x \in A$. Then

cone $A:=\bigcup_{\lambda>0} \lambda A$ (the smallest convex cone containing $A$ ).

qri $A:=\{x \in A \mid \overline{\operatorname{cone}}(A-x)$ is a subspace of $X\}$ (the quasi relative interior of $A$ ).

The infimal convolution plays a central role in our development.

Definition 2.3: Let $f$ and $g$ be closed convex functions on $X$ into the extended reals. Then

$$
(f \square g)(x):=\inf _{y \in X}(f(y)+g(x-y))
$$

is called the inf-convolution.

It is well known that the strict epigraph of the inf-convolution is equal to the setaddition of the strict epigraphs of the individual functions:

$$
\mathrm{epi}_{s}(f \square g)=\mathrm{epi}_{s} f+\mathrm{epi}_{s} g .
$$

Also $\operatorname{dom}(f \square g)=\operatorname{dom} f+\operatorname{dom} g$; epi $f \square g \supseteq$ epi $f+$ epi $g$, and

$$
(f \square g)^{*}=f^{*}+g^{*}
$$


where $f^{*}\left(x^{*}\right)=\sup _{x \in X}\left(\left\langle x, x^{*}\right\rangle-f(x)\right)$ is the Young-Fenchel conjugate of $f$.

Lower semi-continuity of the epi-graphical multi-function $v \mapsto \operatorname{epi}_{s}\left(f_{v} \square g_{v}\right)$ may be deduced from that of its components using the following lemma. We include its derivation due to a lack of a solid reference.

LEMMA 2.1. If $F_{1}(\cdot)$ and $F_{2}(\cdot)$ are multi-functions 1.s.c. at $w$ then $F(v):=$ $F_{1}(v)+F_{2}(v)$ is l.s.c. at $w$.

Proof: We use the classical formulation of l.s.c. (Remark 2.1). Let $N$ be a neighbourhood of a point $y_{0} \in F(w)$. There exist $y_{i} \in F_{i}(w)$ such that $y_{0}=y_{1}+y_{2}$. Take two neighbourhoods $N_{i}$ of $y_{i}$ such that $N_{1}+N_{2} \subseteq N$; there then exist neighbourhoods $V_{i}$ of $w$ for which $F_{i}(v) \cap N_{i} \neq \emptyset$ for all $v \in V_{i}$. Hence for all $v \in V_{1} \cap V_{2}$,

$$
\emptyset \neq F_{1}(v) \cap N_{1}+F_{2}(v) \cap N_{2} \subseteq\left(F_{1}(v)+F_{2}(v)\right) \cap\left(N_{1}+N_{2}\right) \subseteq F(v) \cap N
$$

giving the result.

In [22, Lemma 4.1], it is shown that the epigraphs of the closures $\overline{f_{v} \square g_{v}}$ (in the strong topology on $X$ ) satisfy

$$
\liminf _{v \rightarrow w} \text { epi }\left(\overline{f_{v} \square g_{v}}\right) \supseteq \overline{\operatorname{epi}}\left(f_{w} \square g_{w}\right)
$$

under the condition that $\operatorname{dom} f_{v}^{*} \cap \operatorname{dom} g_{v}^{*} \neq \emptyset$ for $v$ in a neighbourhood of $w$. This result, however, is of limited use here as we are interested in this occurring in the dual space.

We conclude this section with a summary of variational limit notions used in this paper. Let $X$ and $W$ be topological spaces, then for $x \in X, w \in W$, and $\left\{f_{v}\right\}_{v \in W}$ a collection of $\overline{\mathbf{R}}$-valued functions on $X$, define the lower and upper epi-limits by:

$$
\begin{aligned}
& \left(\underset{v \rightarrow w}{\operatorname{e-li}} f_{v}\right)(x):=\sup _{U \in \mathcal{N}(x)} \sup _{V \in \mathcal{N}(w)} \inf _{v \in V} \inf _{y \in U} f_{v}(y), \\
& \left(\underset{v \rightarrow w}{\operatorname{e}-l s} f_{v}\right)(x):=\sup _{U \in \mathcal{N}(x)} \inf _{V \in \mathcal{N}(w)} \sup _{v \in V} \inf _{y \in U} f_{v}(y) .
\end{aligned}
$$

It is well known [20] that these limits correspond to the Kuratowski(-Painlevé) limit of the epi-graph multifunction in the sense that

$$
\begin{aligned}
& \text { epi }\left(\underset{v \rightarrow w}{\operatorname{e}-l s} f_{v}\right)=\underset{v \rightarrow w}{\liminf } \operatorname{epi} f_{v}, \\
& \text { epi }\left(\underset{v \rightarrow w}{\operatorname{e}-l i} f_{v}\right)=\limsup _{v \rightarrow w} \text { epi } f_{v} .
\end{aligned}
$$

These definitions and relations have natural counterparts for nets $\left\{f_{\gamma}\right\}_{\gamma \in I}$ of functions.

DEFINITION 2.4: Let $\left\{f_{v}\right\}_{v \in W}$ be a family of functions. We say that $\left\{f_{v}\right\}_{v \in W}$ is epi-u.s.c. at $w \in W$ if for all $x$ we have

$$
\left(\underset{v \rightarrow w}{\mathrm{e}-\mathrm{ls}} f_{v}\right)(x) \leqslant f_{w}(x)
$$

and epi-l.s.c. if for all $x$

$$
f_{w}(x) \leqslant\left(\underset{v \rightarrow w}{\operatorname{e-li}} f_{v}\right)(x)
$$


Equivalently for an epi-u.s.c. family the epi-graphs of $f_{v}$ are lower Kuratowskiconvergent to epi $f_{\boldsymbol{w}}$

Definition 2.5: A family of functions $\left\{f_{v}\right\}_{v \in W}$ in $\overline{\mathbf{R}}^{X}$ is epi-convergent to a function $f_{w}$ (as $\left.v \rightarrow w\right)$ if it is both epi-u.s.c. and epi-l.s.c. at $w$. equality.

Since $\underset{v \rightarrow w}{\text { e-li }} f_{v} \leqslant \underset{v \rightarrow w}{\mathrm{e}-\mathrm{ls}} f_{v}$ on $X$, the relation defining epi-convergence is in fact an

\section{Semi-Continuities of the Young-Fenchel Conjugate}

In this section we investigate the primal upper topology and dual upper topology whose join with their respective strong lower Kuratowski topologies gives rise respectively to the primal and dual slice topologies. These may be investigated independently without forming slice convergence. First consider the dual space and what would correspond to one half of dual slice convergence.

Definition 3.1: Let $\left\{f_{v}\right\}_{v \in W}$ be a family of functions on $X$ and $\left\{f_{v}^{*}\right\}_{v \in W}$ the family of conjugate functions on $X^{*}$ (for a normed space $X$ ). We denote the boundedweak $^{*}$ upper epi-limit (as $v \rightarrow w$ ) of $\left\{f_{v}^{*}\right\}_{v \in W}$ by

$$
\begin{aligned}
b w^{*}-\limsup _{v \rightarrow w} \text { epi } f_{v}^{*}:=\left\{\left(x^{*}, \alpha\right)\right. & \in X^{*} \times \mathbf{R} \mid \exists \text { nets } v_{\beta} \rightarrow w ;\left(y_{\beta}^{*}, \alpha_{\beta}\right) \in \operatorname{epi} f_{v_{\beta}}^{*} \\
& \text { such that } \left.\alpha_{\beta} \rightarrow \alpha ; y_{\beta}^{*} \text { norm bounded; } y_{\beta}^{*} \stackrel{w^{*}}{\rightarrow} x^{*}\right\} .
\end{aligned}
$$

The above closely resembles the limit-superior of epigraphs, relative to the boundedweak* topology on $X^{*}$ (hence the terminology). The bounded-weak* topology is described in, for example, [16]. Clearly this set recedes to $+\infty$ in the vertical direction and so resembles the epigraph of some function. This prompts us to define

Definition 3.2: For $x^{*} \in X^{*}$,

$$
\left(b w^{*}-\underset{v \rightarrow w}{\operatorname{eli}} f_{v}^{*}\right)\left(x^{*}\right):=\inf \left\{\alpha \in \mathbf{R} \mid\left(x^{*}, \alpha\right) \in b w^{*}-\underset{v \rightarrow w}{\limsup } \operatorname{epi} f_{v}^{*}\right\} .
$$

It then follows that

$$
\operatorname{epi}_{s}\left(b w^{*}-\underset{v \rightarrow-\rightarrow i}{\text { eli }} f_{v}^{*}\right) \subseteq b w^{*}-\limsup _{v \rightarrow w} \text { epi } f_{v}^{*} \subseteq \text { epi }\left(b w^{*}-\underset{v \rightarrow w}{\text { e-li }} f_{v}^{*}\right) .
$$

Thus $b w^{*}-\underset{v \rightarrow w}{\text { e-li }} f_{v}^{*}$ is essentially a variational limit in the sense of [4] or [20]. Analogous definitions can be made for nets $\left\{f_{\gamma}\right\}_{\gamma \in I}$ of functions, that is,

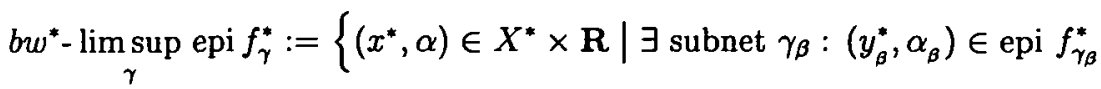

$$
\begin{aligned}
& \text { such that } \left.\alpha_{\beta} \rightarrow \alpha ; y_{\beta}^{*} \text { norm bounded; } y_{\beta}^{*} \stackrel{w^{*}}{\rightarrow} x^{*}\right\} \text {. }
\end{aligned}
$$


with $\left(b w^{*}-\underset{\gamma}{\mathrm{e}-\mathrm{li}} f_{\gamma}^{*}\right)\left(x^{*}\right)$ defined as in (3.1).

We require one half of the so-called slice convergence. The following is the best definition for our purposes although many others are available on modification of existing material (see [6] or [7]).

Definition 3.3: We say $\left\{f_{v}\right\}_{v \in W}$ in $\Gamma(X)$ is upper slice convergent to $f \in \Gamma(X)$ (as $v \rightarrow w$ ) if whenever $v_{\alpha} \rightarrow w$ is a convergent net, $\left\{x_{\alpha}\right\}$ a bounded net in $X$ we have for each $\left(y^{*}, \eta\right) \in \operatorname{epi}_{s} f^{*}$ that $\left.f_{v_{\alpha}}\left(x_{\alpha}\right)\right\rangle\left\langle x_{\alpha}, y^{*}\right\rangle-\eta$ eventually. Denote the set of all $f \in \Gamma(X)$ satisfying the above by $\tau_{s}^{+}-\lim _{v \rightarrow w} f_{v}$. (This choice of notation will be clarified in the next paragraph.) Hereafter we shall denote the pointwise supremum of this class by

$$
\tau_{s}-\underset{v \rightarrow w}{\mathrm{e}-\mathrm{ii}} f_{v}=\sup \left(\tau_{s}^{+}-\lim _{v \rightarrow w} f_{v}\right) \text {. }
$$

Again, analogous definitions follow for nets of functions.

The definition is better understood on noting that $\eta>f^{*}\left(y^{*}\right)$ if and only if $f(x)>$ $\left\langle x, y^{*}\right\rangle-\eta$ for all $x$. A topological way of writing this [7] is to introduce the cylindrical slices $S:=\left\{(x, \alpha) \in X \times \mathbf{R} \mid\|x\| \leqslant \mu ; \alpha=\left\langle x, y^{*}\right\rangle-\eta\right\}$ for any $\left(y^{*}, \eta\right) \in X^{*} \times \mathbf{R}$ and the upper slice topology $\tau_{s}^{+}$on $C(X \times \mathbf{R})$ via the neighbourhood subbasis consisting of the elements

$$
\left(S^{c}\right)^{++}:=\left\{A \in C(X \times \mathbf{R}) \mid A+B(0, \varepsilon) \subseteq S^{c} \text { for some } \varepsilon>0\right\}
$$

for all slices $S$. Then $f \in \tau_{s}^{+}-\lim _{v \rightarrow w} f_{v}$ amounts to epi $f_{v} \in\left(S^{c}\right)^{++}$for all $v$ near $w$ whenever epi $f \in\left(S^{c}\right)^{++}$(in other words, $f_{v} \rightarrow f$ in the topology $\tau_{s}^{+}$). Another subbasis is that generated by $\left(B^{c}\right)^{++}$where $B$ is a closed bounded and non-empty convex subset of $X$. Observe that if $f \in \tau_{s}^{+}-\lim _{v \rightarrow w} f_{v}$ and $g \leqslant f$, then $g \in \tau_{s}^{+}-\lim _{v \rightarrow w} f_{v}$, since epi $g \in\left(B^{c}\right)^{++}$ implies epi $f \in\left(B^{c}\right)^{++}$. This exemplifies the non-Hausdorff nature of $\tau_{s}^{+}$. The lower Kuratowski limit is generated by the neighbourhood subbasis consisting of all sets $N^{-}$ $:=\{B \in C(X \times \mathbf{R}) \mid B \cap N \neq \emptyset\}$ where $N \subseteq X \times \mathbf{R}$ is norm-open. Thus we may speak of a lower topology $\tau^{-}$and the associated lower convergence $\underset{v \rightarrow w}{\operatorname{e}-l s} f_{v} \leqslant f$ by demanding that epi $f \in N^{-}$imply epi $f_{v} \in N^{-}$for all $v$ in a neighbourhood of $w$. The slice topology $\tau_{s}$ is the join of the topologies $\tau_{s}^{+}$and $\tau_{-}$(that is, has as subbasis the union of the subbases of $\tau_{s}^{+}$and $\tau_{-}$).

Recall also the definition of the dual slice topology $\tau_{s}^{*}$ on $X^{*} \times \mathbf{R}$. It is the topology on $C^{*}\left(X^{*} \times \mathbf{R}\right)$ (the class of nonempty weakly* closed convex subsets of $X^{*} \times \mathbf{R}$ ) generated by the subbasis $\left\{V^{-} \mid V\right.$ strongly open in $\left.X^{*} \times \mathbf{R}\right\} \cup\left\{\left(B^{c}\right)^{++} \mid B \in C B^{*}\left(X^{*} \times \mathbf{R}\right)\right\}$, where $C B^{*}\left(X^{*} \times \mathbf{R}\right)$ denotes the set of all bounded nonempty weakly* closed convex subsets of $X^{*} \times \mathbf{R}$. As for the slice topology, the dual topology is expressible as the join of upper and lower topologies, denoted $\tau_{*}^{+}$and $\tau_{*}^{-}$respectively. Note by weak* compactness of $B \in C B^{*}\left(X^{*} \times \mathbf{R}\right)$ that $\left(B^{\mathrm{c}}\right)^{++}=\left(B^{\mathrm{c}}\right)^{+}:=\left\{A \in C^{*}\left(X^{*} \times \mathbf{R}\right) \mid B \cap A=\emptyset\right\}$. The dual slice topology $\tau_{s}^{*}$ on $\Gamma^{*}\left(X^{*}\right)$ is that induced by the map $\Gamma^{*}\left(X^{*}\right) \ni f \mapsto$ epi $f \in C^{*}\left(X^{*} \times \mathbf{R}\right)$. 
LEMMA 3.1. Let $X$ be normed, $W$ topological, and $\left\{f_{v}\right\}_{v \in W}$ be a family of closed proper convex extended-real-valued functions on $X$. Then

$$
\underset{v \rightarrow w}{\mathrm{e}-\mathrm{ls}} f_{v} \geqslant\left(b w^{*}-\underset{v \rightarrow w}{\mathrm{e}-\mathrm{li}} f_{v}^{*}\right)^{*}
$$

and if $V:=\underset{v \rightarrow w}{\mathrm{e}-\mathrm{Is}} f_{v}^{*}$ is in $\Gamma^{*}\left(X^{*}\right)$, then $V^{*} \in \tau_{s}^{+}-\lim _{v \rightarrow w} f_{v}$.

Proof: We first prove the second statement. We show that $V^{*} \in \tau_{s}^{+}-\lim _{v \rightarrow w} f_{v}$. Indeed, let $\left(x^{*}, \eta\right) \in \operatorname{epi}_{s}\left(V^{*}\right)^{*}=\operatorname{epi}_{s} V \neq \emptyset$. Also, let $v_{\gamma} \rightarrow w$, and let $\left(x_{\gamma}\right)$ be a bounded net in $X$. Pick $\beta \in \mathbf{R}$ such that $\eta>\beta>V\left(x^{*}\right)$. As $\left(x^{*}, \beta\right) \in \operatorname{epi} V=\liminf _{v \rightarrow w}$ epi $f_{v}^{*}$, there is a strongly convergent net $\left(x_{\gamma}^{*}, \beta_{\gamma}\right) \rightarrow\left(x^{*}, \beta\right)$ with $f_{v_{\gamma}}^{*}\left(x_{\gamma}^{*}\right) \leqslant \beta_{\gamma}$ eventually. Place $\varepsilon:=\eta-\beta>0$. Then eventually, $\beta_{\gamma}<\beta+\varepsilon / 2$ and $\left\|x_{\gamma}^{*}-x^{*}\right\|<\varepsilon / 2 M$, where $M$ is a bound on the norms of the $x_{\gamma}$. For such $\gamma$ we have from the Fenchel Inequality,

$$
\begin{aligned}
f_{v_{\gamma}}\left(x_{\gamma}\right) & \geqslant\left\langle x_{\gamma}^{*}, x_{\gamma}\right\rangle-f_{v_{\gamma}}^{*}\left(x_{\gamma}^{*}\right)>\left\langle x^{*}, x_{\gamma}\right\rangle-\left(\left\|x_{\gamma}^{*}-x^{*}\right\|\left\|x_{\gamma}\right\|+\beta+\frac{\varepsilon}{2}\right) \\
& >\left\langle x^{*}, x_{\gamma}\right\rangle-(\beta+\varepsilon)=\left\langle x^{*}, x_{\gamma}\right\rangle-\eta .
\end{aligned}
$$

Hence, if $V \in \Gamma^{*}\left(X^{*}\right)$ (so $\left.V^{*} \in \Gamma(X)\right)$ then $V^{*} \in \tau_{s}^{+}-\lim _{v \rightarrow w} f_{v}$.

For this part write $U:=b w^{*}-\underset{v \rightarrow w}{\mathrm{e}-\mathrm{li}} f_{v}^{*}$ and $V:=\underset{v \rightarrow w}{\mathrm{e}-\mathrm{ls}} f_{v}$. We show that $U^{*} \leqslant V$ on $X$. Let $x \in X$. If $V(x)=+\infty$ or $U \equiv+\infty$, (so $U^{*} \equiv-\infty$ ) there is nothing to prove. We then give a proof in the case where $V(x)<+\infty$ and $U$ is not identically $+\infty$ on $X^{*}$. Let $x^{*} \in \operatorname{dom} U$. Let $\alpha, \beta \in \mathbf{R}$ with $\alpha>V(x), \beta>U\left(x^{*}\right)$. Then $(x, \alpha) \in$ epi $V$, $\left(x^{*}, \beta\right) \in$ epi $_{s} U \subseteq b w^{*}$ - $\limsup _{v \rightarrow w}$ epi $f_{v}^{*}$, so there are nets $v_{\gamma} \rightarrow w, x_{\gamma}^{*} \rightarrow x$ (weak*), $\beta_{\gamma} \rightarrow \beta$, with $\left(x_{\gamma}^{*}, \beta_{\gamma}\right) \in$ epi $f_{v_{\gamma}}^{*}$ for each $\gamma$ and the $x_{\gamma}^{*}$ are uniformly norm-bounded. Also, since $(x, \alpha) \in \liminf _{v \rightarrow w}$ epi $f_{v}$, there exists $\left(x_{\gamma}, \alpha_{\gamma}\right)\left(\in\right.$ epi $\left.f_{v_{\gamma}}\right) \rightarrow(x, \alpha)$. For each $\gamma$, $\alpha_{\gamma}+\beta_{\gamma} \geqslant f_{v_{\gamma}}^{*}\left(x_{\gamma}^{*}\right)+f_{v_{\gamma}}^{v \rightarrow w}\left(x_{\gamma}\right) \geqslant\left\langle x_{\gamma}, x_{\gamma}^{*}\right\rangle$ by the Fenchel inequality. As a result, passing to the limit, $\alpha+\beta \geqslant\left\langle x, x^{*}\right\rangle$. (This is permissible as the $x_{\gamma}^{*}$ are norm-bounded and $x_{\gamma} \rightarrow x$ strongly). Rearrange to obtain $\alpha \geqslant\left\langle x, x^{*}\right\rangle-\beta$. Since the $\alpha>V(x)$ and $\beta>U\left(x^{*}\right)$ are arbitrary, we conclude that $V(x) \geqslant\left\langle x, x^{*}\right\rangle-U\left(x^{*}\right)$ and since $x^{*} \in \operatorname{dom} U$ is also arbitrary, it follows that $V(x) \geqslant U^{*}(x)$ as claimed.

The following is motivated by the proof of [4, Theorem 7.5.1].

THEOREM 3.2. Let $X$ be a normed space, $W$ a topological space and $f_{v}(v \in W)$ proper closed convex extended-real-valued functions on $X$. Suppose also that either:

1. the strong epi-limit supremum (as $v \rightarrow w$ ) of $\left\{f_{v}\right\}_{v \in W}$ is not identically $+\infty$, or alternately,

2. for each net $v_{\beta} \rightarrow w$ we have $\tau_{s}$ - e-li $f_{v_{\beta}}$ not identically $+\infty$.

Then for each $f \in \tau_{s}^{+}-\lim _{v \rightarrow w} f_{v}$,

$$
\underset{v \rightarrow w}{\mathrm{e}-\mathrm{ls}} f_{v}^{*} \leqslant f^{*}
$$

where the epi-limit supremum is in the strong topology of $X^{*}$. 
PROOF: Let $f \in \tau_{s}^{+}-\lim _{v \rightarrow w} f_{v}$. Write $V:=\underset{v \rightarrow w}{\operatorname{e-ls}} f_{v}^{*}$. Let $x^{*} \in X^{*}$ and we assume that $V\left(x^{*}\right)>-\infty$, otherwise there is nothing to prove. We show that $\lambda \leqslant f^{*}\left(x^{*}\right)$ whenever $\lambda<V\left(x^{*}\right)$. Suppose $\lambda<V\left(x^{*}\right)$. Then $\left(x^{*}, \lambda\right) \notin$ epi $V=\liminf _{v \rightarrow w}$ epi $f_{v}^{*}$. Then using the alternative definition in Remark 2.2 we have $\limsup _{v \rightarrow w} d\left(\left(x^{*}, \lambda\right)\right.$, epi $\left.f_{v}^{*}\right)>0$ and so there is a net $v_{\beta} \rightarrow w$ and $\varepsilon>0$ for which

$$
(\forall \beta) \quad\left(\left(x^{*}, \lambda\right)+\bar{B}^{*}(0, \varepsilon)\right) \cap \text { epi } f_{v_{\beta}}^{*}=\emptyset
$$

where $\bar{B}^{*}(0, \varepsilon)$ is a ball in the box norm for $X^{*} \times \mathbf{R}$. By the Hahn-Banach Separation Theorem, for each $\beta$ there is $\left\|\left(x_{\beta},-\alpha_{\beta}\right)\right\|=1$ (after rescaling in the box norm) in $X \times \mathbf{R}$ such that

$$
\sup _{\left(x_{\beta}^{*}, \mu_{\beta}\right) \in \operatorname{epi} f_{v_{\beta}}^{*}}\left\langle\left(x_{\beta},-\alpha_{\beta}\right),\left(x_{\beta}^{*}, \mu_{\beta}\right)\right\rangle \leqslant \inf _{\left(y^{*}, \delta\right) \in\left(x^{*}, \lambda\right)+\bar{B}^{*}(0, \varepsilon)}\left\langle\left(x_{\beta},-\alpha_{\beta}\right),\left(y^{*}, \delta\right)\right\rangle .
$$

As $\inf _{\left(y^{*}, \delta\right) \in \bar{B}^{*}(0, \varepsilon)}\left\langle\left(x_{\beta},-\alpha_{\beta}\right),\left(y^{*}, \delta\right)\right\rangle \leqslant-\varepsilon$, it follows that

$$
(\forall \beta)\left(\forall\left(x_{\beta}^{*}, \mu_{\beta}\right) \in \text { epi } f_{v_{\beta}}^{*}\right)\left(\left\langle x_{\beta}, x_{\beta}^{*}\right\rangle-\alpha_{\beta} \mu_{\beta} \leqslant\left\langle x_{\beta}, x^{*}\right\rangle-\alpha_{\beta} \lambda-\varepsilon\right) .
$$

From this follows that $\alpha_{\beta} \geqslant 0$ for such $\beta$ (for if otherwise, take $x_{\beta}^{*} \in \operatorname{dom} f_{\beta}^{*} \neq \emptyset$, and $\mu_{\beta}=f_{v_{\beta}}^{*}\left(x_{\beta}^{*}\right)+k$, and let $k \rightarrow+\infty$ in (3.3) to obtain the contradiction $+\infty \leqslant$ $\left.\left\langle x_{\beta}, x^{*}\right\rangle-\alpha_{\beta} \lambda-\varepsilon\right)$.

Now, if limsup $\alpha_{\beta}>0$, there exist $\delta>0$ and subnet $\alpha_{\beta_{\gamma}}$ such that $\alpha_{\beta_{\gamma}} \geqslant \delta$ for all $\gamma$. Define $\widehat{x}_{\gamma}:=x_{\beta_{\gamma}} / \alpha_{\beta_{\gamma}}$. These have norm bounded above by $1 / \delta$. Dividing (3.3) through by $\alpha_{\beta_{\gamma}}$, letting $x_{\beta_{\gamma}}^{*} \in \operatorname{dom} f_{v_{\beta_{\gamma}}}^{*} \neq \emptyset$ and $\mu_{\beta_{\gamma}}=f_{v_{\beta_{\gamma}}}^{*}\left(x_{\beta_{\gamma}}^{*}\right)$ therein, we get $\left\langle\widehat{x}_{\gamma}, x_{\beta_{\gamma}}^{*}\right\rangle-f_{v_{\beta_{\gamma}}}^{*}\left(x_{\beta_{\gamma}}^{*}\right) \leqslant$ $\left\langle\widehat{x}_{\gamma}, x^{*}\right\rangle-\lambda$, and taking supremum over $x_{\beta_{\gamma}}^{*} \in \operatorname{dom} f_{v_{\beta_{\gamma}}}^{*}$ we have $f_{v_{\beta_{\gamma}}}\left(\widehat{x}_{\gamma}\right) \leqslant\left\langle\widehat{x}_{\gamma}, x^{*}\right\rangle-\lambda$ for all such $\gamma$, where $\widehat{x}_{\gamma}$ is a bounded net. Since $f \in \tau_{s}^{+}-\lim _{v \rightarrow w} f_{v}$, we must have that $\lambda \leqslant f^{*}\left(x^{*}\right)$.

In particular, if $x^{*} \in \operatorname{dom} V$ (so $V\left(x^{*}\right)$ is finite-recall the assumption that $V\left(x^{*}\right)$ $>-\infty)$, we can show that $\liminf _{\beta} \alpha_{\beta}>0$, so $\lambda \leqslant f^{*}\left(x^{*}\right)$ for all $f \in \tau_{s}^{+}-\lim _{v \rightarrow w} f_{v}$ by the previous paragraph. To see this, put $\rho:=V\left(x^{*}\right)-\lambda>0$. As $\left(x^{*}, V\left(x^{*}\right)\right) \in \liminf _{v \rightarrow w}$ epi $f_{v}^{*}$, then for the net $v_{\beta}$ described above, there is a norm-convergent net $\left(x_{\beta}^{*}, \mu_{\beta}\right) \rightarrow\left(x^{*}, V\left(x^{*}\right)\right)$, with $f_{v_{\beta}}\left(x_{\beta}^{*}\right) \leqslant \mu_{\beta}$. Then for all sufficiently large $\beta,\left\|x_{\beta}^{*}-x^{*}\right\| \leqslant \varepsilon / 2$ and $\mu_{\beta} \leqslant V\left(x^{*}\right)+\rho$. We may then use (3.3) to obtain for such $\beta$ :

$$
\begin{gathered}
0 \leqslant\left\langle x_{\beta}, x^{*}-x_{\beta}^{*}\right\rangle+\alpha_{\beta}\left(\mu_{\beta}-\lambda\right)-\varepsilon \leqslant\left\|x^{*}-x_{\beta}^{*}\right\|+\alpha_{\beta}\left(V\left(x^{*}\right)-\lambda+\rho\right)-\varepsilon \\
\leqslant \varepsilon / 2+2 \rho \alpha_{\beta}-\varepsilon
\end{gathered}
$$

which gives $\alpha_{\beta} \geqslant \varepsilon / 4 \rho$, so $\liminf _{\beta} \alpha_{\beta} \geqslant \varepsilon / 4 \rho$. We are then done, for the case of $V\left(x^{*}\right)$ $<+\infty$. 
It only remains to deal with the final case of $V\left(x^{*}\right)=+\infty$. We clearly only need consider the case where $\lim \sup \alpha_{\beta}=0$. We claim the existence of a subnet $v_{\beta_{\gamma}}$ for which there exists a bounded net $\left(q_{\gamma}, \rho_{\gamma}\right)$ with $\left(q_{\gamma}, \rho_{\gamma}\right) \in$ epi $f_{v_{\beta_{\gamma}}}$ for each $\gamma$. In the case that $\underset{v \rightarrow w}{\mathrm{e}-\mathrm{ls}} f_{v}$ is not identically $+\infty$, the claim follows immediately. If instead the assumption is made on the lower slice epi-limit (see the list of hypotheses), we argue this by contradiction as follows: Suppose for all bounded nets $q_{\beta}$ (over the same index set of the $\beta)$ that $\liminf _{\beta} f_{v_{\beta}}\left(q_{\beta}\right)=+\infty$. Then for any $y^{*} \in X^{*}$ and any real $\left.\eta, f_{v_{\beta}}\left(q_{\beta}\right)\right\rangle\left\langle q_{\beta}, y^{*}\right\rangle-\eta$ eventually for any bounded net $\left\{q_{\beta}\right\}$. Hence any proper convex function $f$ will be in $\tau_{s}^{+}-\lim _{\beta} f_{v_{\beta}}$. Consequently, $\tau_{s^{-}}$e-li $f_{v_{\beta}} \equiv+\infty$, a contradiction.

Let $\left(q_{\gamma}, \rho_{\gamma}\right) \in$ epi $f_{v_{\beta_{\gamma}}}$ with $\left\|q_{\gamma}\right\| \leqslant M$ for all $\gamma$, and $\left|\rho_{\gamma}\right| \leqslant M$, as per the above. Let $\mu>0$ be such that $\lambda \leqslant \mu \varepsilon-M\left(1+\left\|x^{*}\right\|\right)$, and multiply (3.3) by $\mu$. Then for all $\gamma$,

$$
\left(\forall\left(x_{\gamma}^{*}, \mu_{\gamma}\right) \in \operatorname{epi~} f_{v_{\beta_{\gamma}}}^{*}\right)\left\langle\mu x_{\beta_{\gamma}}, x_{\gamma}^{*}\right\rangle-\alpha_{\beta_{\gamma}} \mu \mu_{\gamma} \leqslant\left\langle\mu x_{\beta_{\gamma}}, x^{*}\right\rangle-\alpha_{\beta_{\gamma}} \mu \lambda-\mu \varepsilon \text {. }
$$

Also, the Fenchel inequality gives $\left\langle q_{\gamma}, x_{\gamma}^{*}\right\rangle \leqslant f_{v_{\beta_{\gamma}}}^{*}\left(x_{\gamma}^{*}\right)+f_{v_{\beta_{\gamma}}}\left(q_{\gamma}\right)$ and so for such $\gamma$, putting $\mu_{\gamma}=f_{v_{\beta_{\gamma}}}^{*}\left(x_{\gamma}^{*}\right)$ for $x_{\gamma}^{*} \in \operatorname{dom} f_{v_{\beta_{\gamma}}}^{*} \neq \emptyset$, we have (for all $x_{\gamma}^{*} \in \operatorname{dom} f_{v_{\beta_{\gamma}}}^{*}$ )

$$
\begin{aligned}
\left\langle q_{\gamma}+\mu x_{\beta_{\gamma}}, x_{\gamma}^{*}\right\rangle & \leqslant \alpha_{\beta_{\gamma}} \mu f_{v_{\beta_{\gamma}}}^{*}\left(x_{\gamma}^{*}\right)+\left\langle\mu x_{\beta_{\gamma}}, x^{*}\right\rangle+f_{v_{\beta_{\gamma}}}\left(q_{\gamma}\right)+f_{v_{\beta_{\gamma}}}^{*}\left(x_{\gamma}^{*}\right)-\alpha_{\beta_{\gamma}} \mu \lambda-\mu \varepsilon \\
& \leqslant\left(1+\alpha_{\beta_{\gamma}} \mu\right) f_{v_{\beta_{\gamma}}}^{*}\left(x_{\gamma}^{*}\right)+\left\langle\mu x_{\beta_{\gamma}}, x^{*}\right\rangle+\rho_{\gamma}-\alpha_{\beta_{\gamma}} \mu \lambda-\mu \varepsilon .
\end{aligned}
$$

Note that we have $\left\|x_{\beta}\right\| \leqslant 1$. Dividing through the preceding inequality by $1+\alpha_{\beta_{\gamma}} \mu$, and taking the supremum over $x_{\gamma}^{*} \in \operatorname{dom} f_{v_{\beta_{\gamma}}}^{*}$ (then with $f_{v_{\beta}}^{* *}=f_{v_{\beta}}$ )

$$
\begin{aligned}
f_{v_{\beta_{\gamma}}}\left(\frac{q_{\gamma}+\mu x_{\beta_{\gamma}}}{1+\alpha_{\beta_{\gamma}} \mu}\right) & \leqslant \frac{\rho_{\gamma}+\left\langle q_{\gamma}+\mu x_{\beta_{\gamma}}, x^{*}\right\rangle-\left\langle q_{\gamma}, x^{*}\right\rangle-\mu \lambda \alpha_{\beta_{\gamma}}-\mu \varepsilon}{1+\alpha_{\beta_{\gamma}} \mu} \\
& \leqslant\left\langle\frac{q_{\gamma}+\mu x_{\beta_{\gamma}}}{1+\alpha_{\beta_{\gamma}} \mu}, x^{*}\right\rangle-\frac{\mu \lambda \alpha_{\beta_{\gamma}}+\mu \varepsilon-M\left(1+\left\|x^{*}\right\|\right)}{1+\alpha_{\beta_{\gamma}} \mu}
\end{aligned}
$$

Since $\alpha_{\beta} \rightarrow 0$ and since also $\left\|\left(q_{\gamma}+\mu x_{\beta_{\gamma}}\right) /\left(1+\alpha_{\beta_{\gamma}} \mu\right)\right\| \leqslant M+\mu$, from (3.4) it follows on using the definition of the upper slice convergence that

$$
f^{*}\left(x^{*}\right) \geqslant-M\left(1+\left\|x^{*}\right\|\right)+\mu \varepsilon \geqslant \lambda .
$$

This completes the proof.

Similarly, a dual result holds for the bounded-weak* lower epi-limit. Initially we assume the existence of the strong epi-limit of the net of functions on the primal space $X$, but this assumption will be subsequently removed. As preparation, we need a lemma, whose proof will be based on that of $[4$, Theorem 7.5.1]-however, the original argument in the cited reference fails unless existence of the epi-limit is assumed, as we do in the following proof. The point at which the original proof fails will be indicated at the corresponding point in the modified proof below. 
LEMMA 3.3. Let $X$ be a normed space, and $\left\{f_{\beta}\right\}_{\beta \in I}$ a net of proper closed convex extended-real-valued functions on $X$. Suppose also that the strong epi-limit of $\left\{f_{\beta}\right\}_{\beta \in I}$ exists. Also, assume that either:

1. this epi-limit takes a finite value somewhere, or

2. $\quad b w^{*}-\mathrm{e}-\mathrm{li} f_{\beta}^{*}$ is not identically $+\infty$.

Then

$$
\mathrm{e}-\mathrm{ls}_{\beta} f_{\beta}\left(=\underset{\beta}{\mathrm{e}-\mathrm{li}} f_{\beta}\right) \leqslant\left(b w^{*}-\mathrm{e}-\mathrm{li} f_{\beta}^{*}\right)^{*}
$$

Proof: Write $U:=b w^{*}-\underset{\beta}{\mathrm{e}-\mathrm{li}} f_{\beta}^{*}$ and $V:=\underset{\beta}{\mathrm{e}-\mathrm{ls}} f_{\beta}\left(=\underset{\beta}{\mathrm{e}-\mathrm{li}} f_{\beta}\right)$. Assume that $V$ is not identically $-\infty$, otherwise the result follows trivially. Let $x \in X$. It suffices to consider the only non-trivial case-that where $V(x)>-\infty$, and $U\left(x^{*}\right)>-\infty$ for all $x^{*}$ in $X^{*}$. To show that $V(x) \leqslant U^{*}(x)$ we show that $\lambda \leqslant U^{*}(x)$ whenever $\lambda<V(x)$. Suppose $\lambda<V(x)$. Then $(x, \lambda) \notin$ epi $V=\operatorname{limsupepi} f_{\beta}$ and by reasoning analogous to that in the proof of Theorem 3.2 , there are nets $\left\|\left(x_{\beta}^{*},-\alpha_{\beta}\right)\right\|=1$ in $X^{*} \times \mathbf{R}$ such that for some $\varepsilon>0$ and some $\beta_{0}$ (compare with (3.3)),

$$
\left(\forall \beta \succ \beta_{0}\right)\left(\forall\left(x_{\beta}, \mu_{\beta}\right) \in \text { epi } f_{\beta}\right)\left(\left\langle x_{\beta}^{*}, x_{\beta}\right\rangle-\alpha_{\beta} \mu_{\beta} \leqslant\left\langle x_{\beta}^{*}, x\right\rangle-\alpha_{\beta} \lambda-\varepsilon\right) .
$$

From this follows again as in the proof of the preceding Theorem that $\alpha_{\beta} \geqslant 0$ for such $v$. Now, if limsup $\alpha_{\beta}>0$, we have the existence of $\delta>0$ and subnet $\alpha_{\beta_{\gamma}}$ such that $\alpha_{\beta_{\gamma}} \geqslant \delta$ for all $\gamma$. Define $q_{\gamma}^{*}:=x_{\beta_{\gamma}}^{*} / \alpha_{\beta_{\gamma}}$. These have norm bounded above by $1 / \delta$. Dividing (3.5) through by $\alpha_{\beta_{\gamma}}$, letting $x_{\beta_{\gamma}} \in \operatorname{dom} f_{\beta_{\gamma}} \neq \emptyset$ and $\mu_{\beta_{\gamma}}=f_{\beta_{\gamma}}\left(x_{\beta_{\gamma}}\right)$ therein, we get for all $\gamma,\left\langle q_{\gamma}^{*}, x_{\beta_{\gamma}}\right\rangle-f_{\beta_{\gamma}}\left(x_{\beta_{\gamma}}\right) \leqslant\left\langle q_{\gamma}^{*}, x\right\rangle-\lambda$, and taking the supremum over $x_{\beta_{\gamma}} \in \operatorname{dom} f_{\beta_{\gamma}}$, $f_{\beta_{\gamma}}^{*}\left(q_{\gamma}^{*}\right) \leqslant\left\langle q_{\gamma}^{*}, x\right\rangle-\lambda$ for all such $\gamma$. By their norm-boundedness, the $\left\{q_{\gamma}^{*}\right\}$ has a convergent subnet $q_{\gamma_{\eta}}^{*} \stackrel{w^{*}}{\rightarrow} q^{*}$, and so

$$
\left(q_{\gamma_{\eta}}^{*},\left\langle q_{\gamma_{\eta}}^{*}, x\right\rangle-\lambda\right) \stackrel{w^{*}}{\rightarrow}\left(q^{*},\left\langle q^{*}, x\right\rangle-\lambda\right) \in b w^{*}-\limsup _{\beta} \text { epi } f_{\beta}^{*} \subseteq \text { epi } U
$$

Thus $U\left(q^{*}\right) \leqslant\left\langle q^{*}, x\right\rangle-\lambda$, so $\lambda \leqslant U^{*}(x)$. (Note that then $q^{*} \in \operatorname{dom} U$ ).

In particular, if $x \in \operatorname{dom} V$ so $V(x)$ is finite, we can show in an identical fashion to the proof of Theorem 3.2 that $\lim _{\beta} \inf \alpha_{\beta}>0$ and so $\lambda \leqslant U^{*}(x)$ by the previous paragraph and we are done, for the case of $V(x)<+\infty$.

Note that under the current assumption, either, there exists a point where $V$ is finite (at which point we may argue as above to conclude that $\operatorname{dom} U \neq \emptyset$ ), or, no such point exists, in which case it is assumed that $\operatorname{dom} U$ is nonempty (see assumption 2).

It only remains to deal with the final case that $V(x)=+\infty$. Let $q^{*} \in \operatorname{dom} U$ (which we know to be nonempty). Then $U\left(q^{*}\right)$ is finite (for at the beginning of this proof we assume $U>-\infty$ on $\left.X^{*}\right)$, so $\left(q^{*}, U\left(q^{*}\right)+1\right) \in \operatorname{epi}_{s} U \subseteq b w^{*}-\limsup _{\beta}$ epi $f_{\beta}^{*}$ and there is a 
subnet $\beta_{\gamma}$ such that there exists $\left(q_{\gamma}^{*}, \rho_{\gamma}\right) \stackrel{w^{*}}{\rightarrow}\left(q^{*}, U\left(q^{*}\right)+1\right)$, with for some $M,\left\|q_{\gamma}^{*}\right\| \leqslant M$ for all $\gamma$, and $f_{\beta_{\gamma}}^{*}\left(q_{\gamma}^{*}\right) \leqslant \rho_{\gamma}$.

(From this point on, the argument from [4] is questionable, since if assumption (1) holds only for the upper epi-limit, without requiring the existence of the epi-limit-this

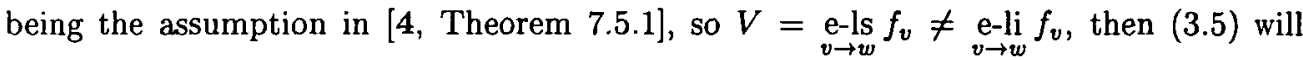
hold merely along some subnet. However the subnet just obtained in regard to $U\left(q^{*}\right)$ has no clear relationship to that along which (3.5) is valid, so information derived on these subnets cannot be merged as they are in the arguments to follow.)

Suppose $\lim \sup _{\gamma} \alpha_{\beta_{\gamma}}>0$. Then restricting (3.5) to the $\beta_{\gamma}$ we may deduce again that $\lambda \leqslant U^{*}(x)$. We are left with the case of $\lim _{\gamma} \alpha_{\beta_{\gamma}}=0$.

Let $\mu>0$ be such that $\left\langle q^{*}, x\right\rangle-U\left(q^{*}\right)+\mu \varepsilon-1 \geqslant \lambda$, and multiply (3.5) by $\mu$. By suitable imitation of arguments in the proof of Theorem 3.2 (compare with the derivation on (3.4)), (3.5) implies

$$
f_{\beta_{\gamma}}^{*}\left(\frac{q_{\gamma}^{*}+\mu x_{\beta_{\gamma}}^{*}}{1+\alpha_{\beta_{\gamma}} \mu}\right) \leqslant \frac{\rho_{\gamma}+\left\langle\mu x_{\beta_{\gamma}}^{*}, x\right\rangle-\mu \lambda \alpha_{\beta_{\gamma}}-\mu \varepsilon}{1+\alpha_{\beta_{\gamma}} \mu} .
$$

By passing to a subnet $\beta_{\gamma_{\eta}}$ whereby $x_{\beta_{\gamma_{\eta}}}^{*}$ has a weak ${ }^{*}$ limit $x^{*}$, then in the limit over $\gamma_{\eta}$, the right-hand side of (3.6) goes to $U\left(q^{*}\right)+1+\left\langle\mu x^{*}, x\right\rangle-\mu \varepsilon$ (since $\alpha_{\beta_{\gamma_{\eta}}} \rightarrow 0$ ), and $\left(q_{\gamma}^{*}+\mu x_{\beta_{\gamma}}^{*}\right) /\left(1+\alpha_{\beta_{\gamma}} \mu\right) \stackrel{w^{*}}{\rightarrow} q^{*}+\mu x^{*}$. Since also $\left\|\left(q_{\gamma}^{*}+\mu x_{\beta_{\gamma}}^{*}\right) /\left(1+\alpha_{\beta_{\gamma}} \mu\right)\right\| \leqslant M+\mu$, it follows from (3.6) that

$$
\left(q^{*}+\mu x^{*}, U\left(q^{*}\right)+1+\left\langle\mu x^{*}, x\right\rangle-\mu \varepsilon\right) \in \operatorname{epi} U .
$$

That is:

$$
U\left(q^{*}+\mu x^{*}\right) \leqslant U\left(q^{*}\right)+\left\langle\mu x^{*}, x\right\rangle-\mu \varepsilon+1
$$

and so

$$
U\left(q^{*}+\mu x^{*}\right)-\left\langle q^{*}+\mu x^{*}, x\right\rangle \leqslant U\left(q^{*}\right)-\left\langle q^{*}, x\right\rangle-\mu \varepsilon+1 .
$$

Therefore

$$
U^{*}(x) \geqslant\left\langle q^{*}+\mu x^{*}, x\right\rangle-U\left(q^{*}+\mu x^{*}\right) \geqslant\left\langle q^{*}, x\right\rangle-U\left(q^{*}\right)+\mu \varepsilon-1 \geqslant \lambda .
$$

This completes the proof.

We now remove the requirement that the epi-limit exists.

THEOREM 3.4. Let $X$ be a normed space, $W$ a topological space; let $\left\{f_{v}\right\}_{v \in W}$ be a family of proper closed convex extended-real-valued functions on $X$. If either:

1. the strong epi-limit supremum $\underset{v \rightarrow w}{\text { e-ls }} f_{v}$ is not identically $+\infty$, and for each $x \in X,\left(\underset{v \rightarrow w}{e-l i} f_{v}\right)(x)>-\infty$; or 
2. for each convergent net $v_{\beta} \rightarrow w$, bw $w^{*}-\underset{\beta}{\mathrm{e}} \mathrm{li} f_{v_{\beta}}^{*}$ is not identically $+\infty$, then,

$$
\underset{v \rightarrow w}{\mathrm{e}-\mathrm{ls}} f_{v}=\left(b w^{*}-\underset{v \rightarrow w}{\mathrm{e}-\mathrm{li}} f_{v}^{*}\right)^{*}
$$

Proof: Let $v_{\beta} \rightarrow w$. By Mrówka's Theorem [7] there is a strongly epi-convergent subnet $f_{v_{\boldsymbol{\beta}_{\gamma}}}$, so that

$$
\underset{v \rightarrow w}{\mathrm{e}-\mathrm{ls}} f_{v} \geqslant \underset{\gamma}{\mathrm{e}-\mathrm{ls}} f_{v_{\beta_{\gamma}}}=\underset{\gamma}{\mathrm{e}-\mathrm{li}} f_{v_{\beta_{\gamma}}} \geqslant \underset{v \rightarrow w}{\mathrm{e}-\mathrm{li}} f_{v} .
$$

(So under assumption (1), the epi-limit of the $f_{v_{\beta_{\gamma}}}$ takes at least one finite value.) By Lemma 3.3 (applied to $f_{v_{\beta_{\gamma}}}$ ),

$$
\underset{\gamma}{\mathrm{e}-\mathrm{ls}} f_{v_{\beta_{\gamma}}} \leqslant\left(b w^{*}-\underset{\gamma}{\mathrm{e}-\mathrm{li}} f_{v_{\beta_{\gamma}}}^{*}\right)^{*} \leqslant\left(b w^{*}-\underset{v \rightarrow w}{\mathrm{e}-\mathrm{li}} f_{v}^{*}\right)^{*} \text {. }
$$

Since this inequality holds for a subnet of each convergent net $v_{\beta} \rightarrow w$, we conclude that

$$
\underset{v \rightarrow w}{\mathrm{e}-\mathrm{ls}} f_{v} \leqslant\left(b w^{*}-\underset{v \rightarrow w}{\mathrm{e}-\mathrm{li}} f_{v}^{*}\right)^{*}
$$

The reverse inequality is the result of Lemma 3.1.

This result extends to non-separable $X$ and non-first-countable parameter space $W$ (hence admitting nets and other generalisations of sequences), some existing results of sequential character $[\mathbf{1}, \mathbf{4}, \mathbf{2 4}]$. These use the sequential analogs of the bounded-weak* lower epi-limits introduced in Definition 3.1. These shall (for sequences of functions $f_{n}^{*}$ ) be denoted by seq- $w^{*}$ - e-li $f_{n \rightarrow \infty}^{*}$. It is easily verified if $X$ is separable (so the weak ${ }^{*}$ topology is metrisable on bounded subsets of $X^{*}$ ), complete, and $W$ is first-countable, that

$$
b w^{*}-\underset{v \rightarrow w}{\mathrm{e}-\mathrm{li}} f_{v}^{*}=\operatorname{seq}-w^{*}-\underset{v \rightarrow w}{\mathrm{e}-\mathrm{li}} f_{v}^{*} .
$$

Now, for the sequence $f_{n} \in \Gamma(X)$, the equality

$$
\underset{n \rightarrow \infty}{\mathrm{e}-\mathrm{ls}} f_{n}=\left(\operatorname{seq}-w^{*}-\underset{n \rightarrow \infty}{\mathrm{e}-\mathrm{li}} f_{n}^{*}\right)^{*}
$$

holds if either:

1. [4, Theorem 7.5.1] $\underset{n \rightarrow \infty}{\mathrm{e}-\mathrm{ls}} f_{n}$ is proper and $X$ is separable; or

2. $\left[1\right.$, Theorem 3.7] $X$ is reflexive, and there is a bounded sequence $x_{n}^{*}$ in $X^{*}$ for which $\sup _{n \in \mathbb{N}} f_{n}^{*}\left(x_{n}^{*}\right)<+\infty$. (Note, however, that by [24, Theorem 1.2] the semicontinuity result implied by (3.7) is in fact valid if the assumption on $X$ is weakened to that of separability, with no completeness assumption. The result in [24] is in fact asserted for any separable metrisable topological linear space, where the boundedness condition on the $x_{n}^{*}$ is understood to be relative to the appropriate topology). 
The proof given in [4], however, contains a gap: the argument therein at one stage attempts to combine information derived on possibly incompatible subsequences; without further assumption, it is not clear that these subsequences have even one point in common, let alone an infinity of such (see also the in-line comment in the proof of Lemma 3.3). This proof is rescued if an assumption on the strong lower epi-limit is appended, as in assumption (1) of our Theorem 3.4.

Case (2) of Theorem 3.4 subsumes the cited result from [1] (or [24]), in the context of normed $X$, since the assumption therein implies assumption (2) of our result. Indeed, given a subnet $n_{\beta}$ of $\mathbf{N}, x_{n_{\beta}}^{*}$ has a weakly* convergent subnet (from the assumed boundedness) with a limit $x^{*}$. Then if $\lambda \in \mathbf{R}$ majorises $\sup _{n \in \mathbb{N}} f_{n}^{*}\left(x_{n}^{*}\right)$, we obtain $\left(x^{*}, \lambda\right) \in b w^{*}-\limsup _{\beta}$ epi $f_{n_{\beta}}$, whence $b w^{*}-\underset{\beta}{-}-1 \mathrm{i} f_{n_{\beta}}$ is not identically $+\infty$.

The following theorem summarises the results obtained so far in this section.

THEOREM 3.5. Let $X$ be a normed space, $W$ a topological space, and $\left\{f_{v}\right\}_{v \in W}$ a family of proper closed convex extended-real-valued functions on $X$. Then:

1. If $f_{v} \stackrel{\tau_{s}^{+}}{\rightarrow} f_{w}$ and either $\underset{v \rightarrow \text {-ls }}{f_{v}} f_{v}$ is not identically $+\infty$, or for each net $v_{\mathcal{\beta}} \rightarrow w$, $\tau_{s}$ - e-li $f_{v_{\beta}}$ is not identically $+\infty$, then $\underset{v \rightarrow w}{\mathrm{e}-\mathrm{ls}} f_{v}^{*} \leqslant f_{w}^{*}$.

2. If $f_{v} \stackrel{\tau_{0}^{-}}{\rightarrow} f_{w}$ (that is, e-Is $f_{v} \leqslant f_{w}$ ), then $f_{w}^{*} \leqslant b w^{*}$ - e-li $f_{v \rightarrow w}^{*}$ on $X^{*}$.

3. If $f_{w}^{*} \leqslant b w^{*}-$ e-li $f_{v \rightarrow w}^{*}$ on $X^{*}$, and either $\underset{v \rightarrow w}{\operatorname{e-ls}} f_{v}$ is not identically $+\infty$ and $\left(\underset{v \rightarrow w}{\mathrm{e}-\mathrm{li}} f_{v}\right)(x)>-\infty$ for all $x \in X$, or for every convergent net $v_{\beta} \rightarrow w$, $b w^{*}$-e-li $f_{v_{\beta}}^{*}$ is not identically $+\infty$, then $f_{w} \geqslant \underset{v \rightarrow w}{\mathrm{e}-l \mathrm{~s}} f_{v}$.

4. If $f_{v}^{*} \stackrel{\tau_{\rightarrow}^{-}}{\rightarrow} f_{w}^{*}$ (that is, e-ls $f_{v \rightarrow w}^{*} \leqslant f_{w}^{*}$ ), and $\underset{v \rightarrow w}{\text { e-ls }} f_{v}^{*} \in \Gamma^{*}\left(X^{*}\right)$, then $f_{v} \stackrel{\tau_{\oplus}^{+}}{\rightarrow} f_{w}$. Proof:

1. From Theorem 3.2 .

2. By Lemma 3.1, $f_{w}^{*} \leqslant\left(\underset{v \rightarrow w}{\text { e-ls }} f_{v}\right)^{*} \leqslant\left(b w^{*}-\underset{v \rightarrow w}{\operatorname{e-li}} f_{v}^{*}\right)^{* *} \leqslant b w^{*}-\underset{v \rightarrow w}{\text { e-li }} f_{v}^{*}$.

3. By Theorem 3.4, e-ls $f_{v \rightarrow w}=\left(b w^{*}-\text { e-li } f_{v \rightarrow w}^{*}\right)^{*} \leqslant f_{w}^{* *}=f_{w}$.

4. By Lemma 3.1, $\left(\underset{v \rightarrow w}{\mathrm{e}-\mathrm{ls}} f_{v}^{*}\right)^{*} \in \tau_{s}^{+}-\lim _{v \rightarrow w} f_{v}$ and hence likewise for $f_{w}$, since $f_{w}=f_{w}^{* *} \leqslant\left(\underset{v}{\operatorname{e}-l s} f_{v}^{*}\right)^{*}$.

As discussed earlier, the slice convergence $f_{v} \rightarrow f_{w}$ is characterised by:

$$
\underset{v \rightarrow w}{\mathrm{e}-l s} f_{v} \leqslant f_{w} \text { and } f_{w} \in \tau_{s}^{+}-\lim _{v \rightarrow w} f_{v}
$$

The result to follow, (combined with the bicontinuity of Young-Fenchel conjugation, with respect to the respective slice topologies on $X$ and $X^{*}$ ), demonstrates that dual slice convergence $g_{v} \rightarrow g_{w}$ (for functions in $\Gamma^{*}\left(X^{*}\right)$ ) has the alternate form:

$$
\underset{v \rightarrow w}{\mathrm{e}-\mathrm{ls}} g_{v} \leqslant g_{w} \leqslant b w^{*}-\underset{v \rightarrow w}{\mathrm{e}-\mathrm{li}} g_{v} \text { on } X^{*} .
$$


(Note since $b w^{*}-\underset{v \rightarrow w}{\text { e-li }} g_{v} \leqslant \underset{v \rightarrow w}{\mathrm{e}-\mathrm{ls}} g_{v}$ always, the inequalities in (3.8) are in fact equalities.)

Corollary 3.6. For functions $f_{v} \in \Gamma(X), f_{v}$ slice-converges to $f_{w}$ if and only if

$$
\underset{v \rightarrow w}{\mathrm{e}-\mathrm{ls}} f_{v}^{*} \leqslant f_{w}^{*} \leqslant b w^{*}-\underset{v \rightarrow w}{\mathrm{e}-\mathrm{li}} f_{v}^{*} .
$$

ProOF: The forward implication is immediate from the first two parts of Theorem 3.5. Conversely, for any net $v_{\beta} \rightarrow w$,

$$
\underset{\beta}{\mathrm{e}-\mathrm{ls}} f_{v_{\beta}}^{*} \leqslant \underset{v \rightarrow w}{\mathrm{e}-\mathrm{ls}} f_{v}^{*} \leqslant f_{w}^{*} \leqslant b w^{*}-\underset{v \rightarrow w}{\mathrm{e}-\mathrm{li}} f_{v}^{*} \leqslant b w^{*}-\underset{\beta}{\mathrm{e}-\mathrm{li}} f_{v_{\beta}}^{*}
$$

and since $b w^{*}-\mathrm{e}-\mathrm{li}_{\beta} f_{v_{\beta}}^{*} \leqslant \mathrm{e}-\mathrm{ls}_{\beta} f_{v_{\beta}}^{*}$, the above forms a chain of equalities. Hence $b w^{*}-\mathrm{e}-\mathrm{li} f_{v_{\beta}}^{*}$ $=f_{w}^{*}$, which is not identically $+\infty$. Consequently $f_{v} \stackrel{\tau_{s}^{-}}{\rightarrow} f_{w}$ by Theorem 3.5 (3). Also, since $\underset{v \rightarrow w}{\text { e-ls }} f_{v}^{*}=f_{w}^{*} \in \Gamma^{*}\left(X^{*}\right)$, we have $f_{v} \stackrel{\tau_{s}^{+}}{\rightarrow} f_{w}$ by Theorem $3.5(4)$.

\section{Sum Theorems for Slice Convergence}

We shall now discuss the passage of slice convergence through addition. Such theorems are closely related to results regarding the passage of the upper (KuratowskiPainlevé) epi-limit through addition. Indeed one only needs to conjoin such a result with Lemma 4.10 (a relatively trivial sum theorem for the upper slice convergence) in order to obtain a result pertaining to slice convergence. Even though Lemma 4.10 is true in normed spaces it requires the coincidence of the weak ${ }^{*}$ and strong closure of a certain convex function in the dual space. This condition may easily be established in a Banach space under a weak assumption (see Remark 4.3) entailed by the hypotheses in the most powerful results on epi-u.s.c. of sums [19], but it is unclear whether one can establish this condition without $X$ being complete. Thus the natural context to frame such results appears to be in a Banach space. Thus we begin assuming $X$ is normed but are often compelled to invoke completeness in stating the main results.

Another approach to such results for slice convergence, to be the concern of this Section, proceeds as follows: Using our duality results of Section 3 we note that the upper slice convergence of $f_{v}+g_{v}$ to $f_{w}+g_{w}$ (as $\left.v \rightarrow w\right)$ dualises to the lower (Kuratowski) epi-convergence of $\overline{f_{v}^{*} \square g_{v}^{*}} \boldsymbol{w}^{*}$ to $\overline{f_{w}^{*} \square g_{w}^{*}} w^{*}$. This in turn relates to the lower Kuratowski convergence of the sum of the (strict) epigraphical multifunctions $v \mapsto$ epi $f_{v}^{*}$ and $v \mapsto$ epi $g_{v}^{*}$, which may be established quite generally. Thus in order to establish slice convergence of $\left\{f_{v}+g_{v}\right\}_{v \in W}$ we may utilise the continuity results of section 3 to reduce the problem to one of establishing the dual upper slice convergence of $\left\{\bar{f}_{v}^{*} \square g_{v}^{*}\right\}_{v \in W^{*}}$. The dual upper slice topology is, similarly, related to the upper Kuratowski convergence of epigraphs relative to the bounded-weak ${ }^{*}$ topology, an observation which motivates us to investigate the boundedness of the sets given in Definition 4.1. These considerations appear to be fundamental to the approach taken here. 
Definition 4.1: Following [3], define for $K \in \mathbf{R}$,

$$
H_{K}:=\left\{\left(x_{1}^{*}, x_{2}^{*}\right) \in X^{*} \times X^{*} \mid f^{*}\left(x_{1}^{*}\right)+g^{*}\left(x_{2}^{*}\right) \leqslant K,\left\|x_{1}^{*}+x_{2}^{*}\right\| \leqslant K\right\},
$$

and for functions $f_{v}, g_{v}(v \in W)$,

$$
H_{K}(v):=\left\{\left(x^{*}, y^{*}\right) \in X^{*} \times X^{*} \mid f_{v}^{*}\left(x^{*}\right)+g_{v}^{*}\left(y^{*}\right) \leqslant K,\left\|x^{*}+y^{*}\right\| \leqslant K\right\} .
$$

Lemma 4.1. Let $f$ and $g$ be in $\Gamma(X)$ for a Banach space $X$, such that $H_{K}$ is bounded for each $K \in \mathbf{R}$. Then $f^{*} \square g^{*} \in \Gamma^{*}\left(X^{*}\right)$.

Proof: For the weak ${ }^{*}$ lower-semicontinuity, it suffices by the Krein-Šmulian Theorem [14], to prove that $\left\{f^{*} \square g^{*} \leqslant \mu\right\} \cap \bar{B}^{*}(0, M)$ is weak* closed for each $\mu, M$.

Let $x_{\beta}^{*} \stackrel{w^{*}}{\rightarrow} x^{*}$ with $x_{\beta}^{*} \in\left\{f^{*} \square g^{*} \leqslant \mu\right\} \cap \bar{B}^{*}(0, M)$ for each $\beta$, and let $\varepsilon>0$. Then $\left\|x^{*}\right\| \leqslant M$ by the weak* lower-semicontinuity of the dual norm. Also, for each $\beta$, there is $y_{\beta}^{*} \in X^{*}$ such that

$$
f^{*}\left(x_{\beta}^{*}-y_{\beta}^{*}\right)+g^{*}\left(y_{\beta}^{*}\right) \leqslant \mu+\varepsilon .
$$

Then $\left(x_{\beta}^{*}-y_{\beta}^{*}, y_{\beta}^{*}\right) \in H_{K}$ where $K=\max \{\mu+\varepsilon, M\}$, so $y_{\beta}^{*}$ is bounded, and passing to a subnet, we may assume $y_{\beta}^{*} \stackrel{w^{*}}{\rightarrow} y^{*}$ for some $y^{*}$. Then, since $x_{\beta}^{*} \stackrel{w^{*}}{\rightarrow} x^{*}$, we conclude

$$
\begin{aligned}
\left(f^{*} \square g^{*}\right)\left(x^{*}\right) & \leqslant f^{*}\left(x^{*}-y^{*}\right)+g^{*}\left(y^{*}\right) \\
& \leqslant \liminf _{\beta}^{*}\left(x_{\beta}^{*}-y_{\beta}^{*}\right)+\liminf _{\beta} g^{*}\left(y_{\beta}^{*}\right) \\
& \leqslant \liminf _{\beta}\left(f^{*}\left(x_{\beta}^{*}-y_{\beta}^{*}\right)+g^{*}\left(y_{\beta}^{*}\right)\right) \leqslant \mu+\varepsilon,
\end{aligned}
$$

and hence, on letting $\varepsilon \rightarrow 0$, that the level-set of $f^{*} \square g^{*}$ is weakly* closed.

To verify the properness, suppose to the contrary that $\left(f^{*} \square g^{*}\right)\left(x^{*}\right)=-\infty$ for some $x^{*}$. Then $\lim _{\beta}\left(f^{*}\left(x^{*}-y_{\beta}^{*}\right)+g^{*}\left(y_{\beta}^{*}\right)\right)=-\infty$ for some net $y_{\beta}^{*}$, and since $\left(x^{*}-y_{\beta}^{*}, y_{\beta}^{*}\right) \in H_{\left\|x^{*}\right\|}$, we have $y_{\beta}^{*} \stackrel{w^{*}}{\rightarrow} y^{*}$ on taking a subnet. Then

$$
\begin{aligned}
-\infty<f^{*}\left(x^{*}-y^{*}\right)+g^{*}\left(y^{*}\right) & \leqslant \liminf _{\beta} f^{*}\left(x^{*}-y_{\beta}^{*}\right)+\liminf _{\beta} g^{*}\left(y_{\beta}^{*}\right) \\
& \leqslant \liminf _{\beta}\left(f^{*}\left(x^{*}-y_{\beta}^{*}\right)+g^{*}\left(y_{\beta}^{*}\right)\right)=-\infty,
\end{aligned}
$$

a contradiction.

The following lemma provides bounds which will be of use in the next Theorem.

LEMMA 4.2. Let $\left\{f_{v}\right\}_{v \in W}$ be a family of proper closed convex extended-realvalued functions on a normed space $X$. Suppose that $\left\{f_{v}\right\}_{v \in W}$ is strongly epi-u.s.c. with respect to $f_{w}$. Then for each $M>0$,

$$
\left(\exists V^{\prime} \in \mathcal{N}(w)\right)(\exists \mu \in \mathbf{R})\left(\forall v \in V^{\prime}\right)\left(\forall\left\|x^{*}\right\| \leqslant M\right)\left(f_{v}^{*}\left(x^{*}\right) \geqslant \mu\right) .
$$


Proof: Let $M>0$ and suppose the assertion false. Then there are nets $v_{\beta} \rightarrow$ $w,\left\|x_{\beta}^{*}\right\| \leqslant M$ such that $\lim _{\beta} f_{v_{\beta}}^{*}\left(x_{\beta}^{*}\right)=-\infty$. By taking a weakly* convergent subnet $x_{\beta_{\gamma}}^{*} \rightarrow x^{*}$, it follows for any real $\lambda$, that $\left(x^{*}, \lambda\right) \in b w^{*}$ - $\limsup _{v \rightarrow w}$ epi $f_{v}^{*} \subseteq$ epi $\left(b w^{*}-\right.$ e-li $\left.f_{v \rightarrow w}^{*}\right)$, so $\left(b w^{*}-\underset{v \rightarrow w}{\text { e-li }} f_{v}^{*}\right)\left(x^{*}\right)=-\infty$, which in turn implies via Lemma 3.1 , that $f_{w} \geqslant \underset{v \rightarrow w}{\text { e-ls }} f_{v}$ $\geqslant\left(b w^{*}-\underset{v \rightarrow w}{\mathrm{e}-\mathrm{li}} f_{v}^{*}\right)^{*} \equiv+\infty$, contradicting the properness of $f_{w}$.

We now state a preliminary version of our main result, which will be the startingpoint for all subsequent investigations in this Section.

THEOREM 4.3. Let $\left\{f_{v}\right\}_{v \in W}$ and $\left\{g_{v}\right\}_{v \in W}$ be families of proper closed convex extended-real-valued functions on a normed space $X$, which are slice convergent (as $v \rightarrow w)$ to $f_{w}$ and $g_{w}$ respectively. Assume also the following:

1. $f_{v}^{*} \square g_{v}^{*} \in \Gamma^{*}\left(X^{*}\right)$ for all $v$ near $w$;

2. for each $K \geqslant 0$, there is $V \in \mathcal{N}(w)$ for which $\bigcup_{v \in V} H_{K}(v)$ is bounded.

Then $\left\{f_{v}+g_{v}\right\}_{v \in W}$ is slice convergent to $f_{w}+g_{w}$ as $v \rightarrow w$.

ProOF: From the bicontinuity of the Young-Fenchel transform (with respect to the slice topology), and the relation $f_{v}+g_{v}=\left(f_{v}^{*} \square g_{v}^{*}\right)^{*}$, it suffices to prove the dual slice convergence of $f_{v}^{*} \square g_{v}^{*}$ to $f_{w}^{*} \square g_{w}^{*}$. From Corollary 3.6,v $\mapsto$ epi $f_{v}^{*}$ and $v \mapsto$ epi $g_{v}^{*}$ are both strongly lower-semicontinuous at $v=w$. Then

$$
\begin{aligned}
\operatorname{epi}_{s} f_{w}^{*} \square g_{w}^{*} & \subseteq \operatorname{epi} f_{w}^{*}+\text { epi } g_{w}^{*} \\
& \subseteq \liminf _{v \rightarrow w}\left(\text { epi } f_{v}^{*}+\text { epi } g_{v}^{*}\right) \text { by Lemma } 2.1 \\
& \subseteq \liminf _{v \rightarrow w} \operatorname{epi} f_{v}^{*} \square g_{v}^{*}
\end{aligned}
$$

and since the latter set is strongly closed,

$$
\text { epi } f_{w}^{*} \square g_{w}^{*} \subseteq \overline{\operatorname{epi}_{s} f_{w}^{*} \square g_{w}^{*}} \subseteq \liminf _{v \rightarrow w} \text { epi } f_{v}^{*} \square g_{v}^{*},
$$

that is, $v \mapsto$ epi $f_{v}^{*} \square g_{v}^{*}$ is strongly lower-semicontinuous at $v=w$.

The remaining task (by Corollary 3.6), is to show that

$$
f_{w}^{*} \square g_{w}^{*} \leqslant b w^{*}-\underset{v \rightarrow w}{\mathrm{e}-\mathrm{li}_{v}} f_{v}^{*} \square g_{v}^{*},
$$

or equivalently,

$$
b w^{*}-\underset{v \rightarrow w}{\limsup } \operatorname{epi} f_{v}^{*} \square g_{v}^{*} \subseteq \text { epi } f_{w}^{*} \square g_{w}^{*} \text {. }
$$

Let $\left(x^{*}, \alpha\right) \in b w^{*}$ - limsupepi $f_{v}^{*} \square g_{v}^{*}$. Then there is $M>0$, and nets $v_{\beta} \rightarrow w,\left(x_{\beta}^{*}, \alpha_{\beta}\right)$ $\in \bar{B}^{*}(0, M) \cap$ epi $f_{v_{\beta}}^{*} \square g_{v_{\beta}}^{*}$ with the latter weakly* convergent to $\left(x^{*}, \alpha\right)$. Redefining the index set for the net, we may, without losing generality, assume that $\left(x_{\beta}^{*}, \alpha_{\beta}\right)$ is in the strict epigraph of $f_{v_{\beta}}^{*} \square g_{v_{\beta}}^{*}$. 
For each $\beta$, we then have the decomposition

$$
\left(x_{\beta}^{*}, \alpha_{\beta}\right)=\left(x_{1_{\beta}}^{*}, \alpha_{1_{\beta}}\right)+\left(x_{2_{\beta}}^{*}, \alpha_{2_{\beta}}\right) \in\left(\text { epi } f_{v_{\beta}}^{*}+\operatorname{epi} g_{v_{\beta}}^{*}\right) \cap B^{*}(0, M),
$$

from which follows that $\left(x_{1_{\beta}}^{*}, x_{2_{\beta}}^{*}\right) \in H_{M}\left(v_{\beta}\right)$ and hence are eventually bounded, from assumption (2). This implies likewise for the $\alpha_{1_{\beta}}$ and $\alpha_{2_{\beta}}$. Indeed, if $\left\|\left(x_{1_{\beta}}^{*}, x_{2_{\beta}}^{*}\right)\right\| \leqslant M^{\prime}$ for some $M^{\prime}$, then from Lemma 4.2 , there is $\mu \in \mathbf{R}$ such that eventually $\alpha_{1_{\beta}} \geqslant f_{v_{\beta}}^{*}\left(x_{1_{\beta}}^{*}\right) \geqslant \mu$ and $\alpha_{2_{\beta}} \geqslant g_{v_{\beta}}^{*}\left(x_{2_{\beta}}^{*}\right) \geqslant \mu$, and since $\alpha_{1_{\beta}}+\alpha_{2_{\beta}} \rightarrow \alpha \in \mathbf{R}$, it follows that they are also bounded above.

Hence, from the boundedness of $\left\|x_{i_{\beta}}^{*}\right\|,\left|\alpha_{i_{\beta}}\right|(i=1,2)$, we may extract convergent subnets

$$
x_{1_{\beta_{\gamma}}}^{*} \stackrel{w^{*}}{\rightarrow} x_{1}^{*}, \quad x_{2_{\beta_{\gamma}}^{*}}^{*} \stackrel{w^{*}}{\rightarrow} x_{2}^{*}, \quad \alpha_{1_{\beta_{\gamma}}} \rightarrow \alpha_{1}, \quad \alpha_{2_{\beta_{\gamma}}} \rightarrow \alpha_{2},
$$

so

$$
\begin{aligned}
\left(x^{*}, \alpha\right) & =w^{*}-\lim _{\gamma}\left(\left(x_{1_{\beta_{\gamma}}^{*}}^{*}, \alpha_{1_{\beta_{\gamma}}}\right)+\left(x_{2_{\beta_{\gamma}}}^{*}, \alpha_{\beta_{\beta_{\gamma}}}\right)\right)=\left(x_{1}^{*}, \alpha_{1}\right)+\left(x_{2}^{*}, \alpha_{2}\right) \\
& \in b w^{*}-\limsup _{v \rightarrow w} \text { epi } f_{v}^{*}+b w^{*}-\underset{v \rightarrow w}{\limsup \text { epi } g_{v}^{*}} \\
& \subseteq \operatorname{epi~}\left(b w^{*} \text { - e-li } f_{v \rightarrow w}^{*}\right)+\operatorname{epi}\left(b w^{*}-\text { e-li } g_{v \rightarrow w}^{*}\right) \\
& \subseteq \operatorname{epi~} f_{w}^{*}+\operatorname{epi} g_{w}^{*} \quad \text { from the slice convergence of } f_{v} \text { and } g_{v} \text { (Corollary 3.6) } \\
& \subseteq \operatorname{epi~} f_{w}^{*} \square g_{w}^{*} .
\end{aligned}
$$

hence proving (4.2).

From this we can recover a result alternately deducible from a theorem of Penot [19] (valid on a normed space) on the epi-upper-semicontinuity of sums.

COROLlary 4.4. Let $\left\{f_{v}\right\}_{v \in W}$ and $\left\{g_{v}\right\}_{v \in W}$ be families of proper closed convex extended-real-valued functions on a Banach space $X$, which are slice convergent (as $v \rightarrow w)$ to $f_{w}$ and $g_{w}$ respectively, such that for some $\mu>0, \delta>0$

$$
B(0, \delta) \subseteq\left\{f_{v} \leqslant \mu\right\} \cap B(0, \mu)-\left\{g_{v} \leqslant \mu\right\} \cap B(0, \mu)
$$

for all $v$ in a neighbourhood $V$ of $w$. Then $\left\{f_{v}+g_{v}\right\}_{v \in W}$ is slice convergent to $f_{w}+g_{w}$ as $v \rightarrow w$.

Proof: To verify condition (4.3) in Theorem 4.3, we follow an argument in [3]. Indeed, it suffices, in view of the Uniform Boundedness Principle, to check that for each element $(x, y)$ of $X \times X$ there is a constant $C(x, y)$ such that for all $\left(x^{*}, y^{*}\right) \in \bigcup_{v \in V} H_{K}(v)$, we have $\left\langle x^{*}, x\right\rangle+\left\langle y^{*}, y\right\rangle \leqslant C(x, y)$. Now, $x-y \in \lambda B(0, \delta)$ for some $\lambda>0$, so that for each $v \in V, x-y=\lambda\left(x_{v}-y_{v}\right)$ with $f_{v}\left(x_{v}\right) \leqslant \mu, g_{v}\left(y_{v}\right) \leqslant \mu$ and $\left\|x_{v}\right\| \leqslant \mu,\left\|y_{v}\right\| \leqslant \mu$. Then, since $\left(x^{*}, y^{*}\right) \in H_{K}(v)$ for some $v$,

$$
\begin{aligned}
\left\langle x^{*}, x\right\rangle+\left\langle y^{*}, y\right\rangle & =\lambda\left\langle x^{*}, x_{v}\right\rangle+\lambda\left\langle y^{*}, y_{v}\right\rangle+\left\langle x^{*}+y^{*}, y-\lambda y_{v}\right\rangle \\
& \leqslant \lambda\left(f_{v}^{*}\left(x^{*}\right)+f_{v}\left(x_{v}\right)+g_{v}^{*}\left(y^{*}\right)+g_{v}\left(y_{v}\right)\right)+\left\|x^{*}+y^{*}\right\| \cdot\left\|y-\lambda y_{v}\right\| \\
& \leqslant \lambda(K+2 \mu)+K(\|y\|+\lambda \mu):=C(x, y) .
\end{aligned}
$$


Hence $\bigcup_{v \in V} H_{K}(v)$ is bounded for each $K$, which, along with Lemma 4.1 , implies all the conditions of the Theorem.

This result itself subsumes an earlier result of Lahrache (see [7]).

Corollary 4.5. In particular, the above result follows if $\left\{g_{v}\right\}_{v \in W}$ is uniformly bounded above on a neighbourhood of some point of $\operatorname{dom} f_{w}$.

ProOF: The epi-u.s.c. of $\left\{f_{v}\right\}_{v \in W}$ at $w$ along with the assumption implies (4.3).

We now briefly digress, to discuss the derivation of Corollary 4.4 from the cited result of [19]. This result asserts the epi-u.s.c. of the sum from that of its components, under the condition (4.3), and is valid in general normed space. On combination with Lemma 4.10 (a sum theorem for the upper slice topology) it yields the slice convergence of the sum (if the components slice-converge) subject to (4.3) and the weak* closure condition

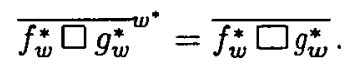

From Remark 4.3 following Theorem $4.11,0 \in \operatorname{int}\left(\operatorname{dom} f_{w}-\operatorname{dom} g_{w}\right)$ implies, if $X$ is Banach, that $f_{w}^{*} \square g_{w}^{*} \in \Gamma^{*}\left(X^{*}\right)$ which in turn implies (4.4). However, if $X$ is not complete, it is not immediately obvious that $0 \in \operatorname{int}\left(\operatorname{dom} f_{w}\right.$-dom $g_{w}$ ) indeed entails (4.4). If it did, then [19] would yield the assertion of Corollary 4.4 in a general normed space. However, the above considerations suggest (but do not prove, of course) that completeness of $X$ seems necessary for slice convergence in this situation. We shall not further pursue the resolution of this issue.

Note that the assumption (4.3) acts simultan eously on both $\left\{f_{v}\right\}_{v \in W}$ and $\left\{g_{v}\right\}_{v \in W}$, whereas that of Corollary 4.5 is only on one of these families. (This condition however, is so strong that in this context, it implies (4.3)). Guided by the form of this latter assumption, we shall use Theorem 4.3 to seek conditions that can be isolated to one of $\left\{f_{v}\right\}_{v \in W}$ or $\left\{g_{v}\right\}_{v \in W}$, but not so strong that the results obtained are manifestly subsumed by Corollary 4.4 .

The sufficient conditions we shall derive rely on recession properties of level-sets. We therefore begin with a review of some relevant concepts.

Recall for any closed convex subset $A$ of a topological linear space $X$, the recession cone $\operatorname{rec} A$ is defined by

$$
\operatorname{rec} A:=\bigcap_{\lambda>0} \lambda(A-a)
$$

for any fixed $a \in A$. It is easily seen to be independent of the particular choice of $a$, so is well-defined. Further, we have the equivalent form

$$
\operatorname{rec} A:=\{x \in X \mid x+A \subseteq A\}
$$


Evidently $\operatorname{rec} A$ is a closed convex cone.

The recession function [21] $f 0^{+}$of the closed convex function $f$ is the (closed convex) function with epigraph rec (epi $f$ ). An explicit formula for this function (when $f$ is proper) is $\left(f 0^{+}\right)(y)=\lim _{\lambda \rightarrow \infty}(f(x+\lambda y)-f(x)) / \lambda$ and is independent of the choice of $x \in \operatorname{dom} f$. Thus clearly $f 0^{+}$is positively homogeneous. If $f$ is also proper then all nonempty levelsets $\{f \leqslant \alpha\}$ have the same recession cones which at times will be $\operatorname{denoted}$ by $\operatorname{rec} f$. Indeed, by definition $\left(f 0^{+}\right)(y) \leqslant 0$ if and only if the recession cone for epi $f$ contains $(y, 0)$ and so $f(x+\lambda y) \leqslant f(x) \leqslant \alpha$ for all $\lambda \geqslant 0$ and any $x \in\{f \leqslant \alpha\}$ or $x+\lambda y \in\{f \leqslant \alpha\}$. Hence $y \in \operatorname{rec}\{f \leqslant \alpha\}$ if and only if $\left(f 0^{+}\right)(y) \leqslant 0$. Finally we note that if $f \in \Gamma(X)$, then the support function to $\operatorname{dom} f$ is the recession function $f^{*} 0^{+}$of $f^{*}$, and the support function to $\operatorname{dom} f^{*}$ is the recession function $f 0^{+}$of $f[21$, Corollary 3D].

LEMMA 4.6. Let $g \in \Gamma(X)$. Then $(\mathrm{rec} g)^{\circ}=\overline{\operatorname{cone}}^{\omega^{*}}$ dom $g^{*}$.

Proof: From the relations of the previous paragraph, $\operatorname{rec} g=\left\{g 0^{+} \leqslant 0\right\}=\left\{S\left(\operatorname{dom} g^{*}, \cdot\right) \leqslant 0\right\}=\left\{S\left(\overline{\operatorname{cone}^{w^{*}}} \operatorname{dom} g^{*}, \cdot\right) \leqslant 0\right\}={ }^{o}\left(\overline{\operatorname{cone}}^{w^{*}} \operatorname{dom} g^{*}\right)$. Taking polars of both sides gives the result.

LEMMA 4.7. Suppose $f$ and $g$ are proper closed convex functions on a normed space $X$. Then for any $y^{*} \in \operatorname{dom} f^{*}+\operatorname{dom} g^{*}$,

$$
(\overline{\operatorname{cone}}(\operatorname{dom} f-\operatorname{dom} g))^{o}=\operatorname{rec}\left[f^{*}+g^{*}\left(y^{*}-\cdot\right)\right] \text {. }
$$

Proof: Define $\widehat{g} \in \Gamma(X)$ by $\widehat{g}(x)=g(-x)+\left\langle y^{*}, x\right\rangle$. Then if $h:=f \square \hat{g}$, we have $h^{*}=f^{*}+\widehat{g}^{*}=f^{*}+g^{*}\left(y^{*}-.\right) \in \Gamma^{*}\left(X^{*}\right)$ since $y^{*} \in \operatorname{dom} f^{*}+\operatorname{dom} g^{*}$. Thus the closure $\bar{h}$ of $h$ is in $\Gamma(X)$, and so $S(\operatorname{dom} \bar{h}, \cdot)=\bar{h}^{*} 0^{+}$, from which it follows that $S(\operatorname{dom} h, \cdot)=h^{*} 0^{+}$, (since $\operatorname{dom} h \subseteq \operatorname{dom} \bar{h} \subseteq \overline{\operatorname{dom} h}$, so that $S\left(\operatorname{dom} \bar{h}, x^{*}\right)=S\left(\operatorname{dom} h, x^{*}\right)$ by continuity of $\left.x^{*}\right)$. As a result,

$$
\begin{aligned}
(\text { cone }(\operatorname{dom} f-\operatorname{dom} g))^{\circ} & =(\text { cone dom } h)^{\circ} \\
& =\{S(\operatorname{dom} h, \cdot) \leqslant 0\}=\left\{h^{*} 0^{+} \leqslant 0\right\}=\operatorname{rec} h^{*} .
\end{aligned}
$$

REMARK 4.1. The hypothesis $\overline{\operatorname{cone}}(\operatorname{dom} f-\operatorname{dom} g)=X$ in fact implies that rec $H_{K}$ $=\{0\}$. Indeed, let $\left(x^{*}, y^{*}\right) \in H_{K}$ and $\left(z_{1}^{*}, z_{2}^{*}\right) \in \operatorname{rec} H_{K}$. Then for all $\lambda \geqslant 0, f^{*}\left(x^{*}+\lambda z_{1}^{*}\right)$ $+g^{*}\left(y^{*}+\lambda z_{2}^{*}\right) \leqslant K$ and $\left\|x^{*}+y^{*}+\lambda\left(z_{1}^{*}+z_{2}^{*}\right)\right\| \leqslant K$. The latter implies that $z_{1}^{*}+z_{2}^{*}=0$, so placing $z^{*}:=z_{1}^{*}=-z_{2}^{*}$ in the former, we obtain that $z^{*} \in \operatorname{rec}\left[f^{*}\left(x^{*}+\cdot\right)+g^{*}\left(y^{*}-\cdot\right)\right]=$ $\operatorname{rec}\left[f^{*}+g^{*}\left(y^{*}+x^{*}-\cdot\right)\right]=\{0\}$ by the Lemma, since $x^{*}+y^{*} \in \operatorname{dom} f^{*}+\operatorname{dom} g^{*}$.

In finite dimensions the knowledge that the recession cone of a convex set is a singleton is sufficient to deduce boundedness of the set. This generally fails in infinite 
dimensions. From Theorem 4.3, we want to establish the boundedness, uniformly in $v$, of the sets $H_{K}(v)$. If we merely assume $\overline{c o n e}$ (dom $\left.f_{v}-\operatorname{dom} g_{v}\right)=X$ at each $v$, then $H_{K}(v)$ has no recession directions by Lemma 4.7 , but as noted earlier, this is insufficient to ensure even the boundedness of any one such set, much less a bound uniform in $v$.

For this, some kind of (weak*) local compactness (uniformly in $v$ ) will be needed. This will permit an argument by contradiction in that if there is no such uniform boundedness, then unbounded nets in these sets can be constructed, which, upon normalisation, converge weak* to a non-zero member of $H_{K}(w)$, contradicting the assumption $\overline{\text { cone }}\left(\operatorname{dom} f_{w}-\operatorname{dom} g_{w}\right)=X$ at $w$.

The following definition and lemma will furnish the required constraint qualification.

Definition 4.2: For $h_{1}, . ., h_{m}$ in $X$,

$$
K\left(h_{1}, . ., h_{m}\right):=\left\{x^{*} \in X^{*}\left|\left\|x^{*}\right\| \leqslant \max _{j=1, \ldots, m}\right|\left\langle x^{*}, h_{j}\right\rangle \mid\right\}
$$

Clearly this forms a weak* ${ }^{*}$ closed convex cone. Further, it is weak* locally compact [18].

LEMMA 4.8. Let $\left\{g_{v}\right\}_{v \in W}$ be a collection of functions in $\Gamma(X)$. Then the condition

$$
(\exists r>0 ; H \text { compact in } X ; V \in \mathcal{N}(w))(\forall v \in V)\left(B(0, r) \subseteq \operatorname{rec} g_{v}-H\right)
$$

holds if and only if there exist $h_{1}, \ldots, h_{m}$ in $X$ such that

$$
\operatorname{dom} g_{v}^{*} \subseteq K\left(h_{1}, . ., h_{m}\right)
$$

for all $v$ in a neighbourhood of $w$.

REMARK 4.2. The condition (4.5) is equivalent to $\left\{\text { rec } g_{v}\right\}_{v \in W}$ being compactly epiLipschitz at 0 , but uniformly in $v$ near $w$. (For definition of "compactly epi-Lipschitz" see $[10,17])$. Also, as shown in $[10$, Lemma 2], the content of $(4.5)$ is unaltered if $H$ is taken to be in addition, finite-dimensional.

ProOF: (The argument to follow is a specialisation of one used in [17].) Assume (4.5). Let $0<\varepsilon<r$ be such that $H \subseteq \bigcup_{i=1}^{m}\left(k_{i}+B(0, \varepsilon)\right)$ for some $k_{i} \in H$ (from compactness of $H)$. Place $h_{i}:=k_{i} /(r-\varepsilon)$. Let $v \in V$ and $x^{*} \in \overline{\operatorname{cone}}^{w^{*}} \operatorname{dom} g_{v}^{*}=\left(\operatorname{rec} g_{v}\right)^{o}$. Then $\left\langle x^{*}, x\right\rangle \leqslant 0$ for all $x \in \operatorname{rec} g_{v}$. If $\|u\| \leqslant 1$, then $r u \in \operatorname{rec} g_{v}-H$, so $r u+h \in \operatorname{rec} g_{v}$ for some $h \in H$. Then since $\left\langle x^{*}, r u+h\right\rangle \leqslant 0$, we obtain

$$
\left\langle x^{*}, r u\right\rangle \leqslant-\left\langle x^{*}, h\right\rangle \leqslant\left|\left\langle x^{*}, h\right\rangle\right| \leqslant\left|\left\langle x^{*}, k_{i}\right\rangle\right|+\varepsilon\left\|x^{*}\right\|
$$

for some $i$. Hence, since $u$ is arbitrary,

$$
\left\|x^{*}\right\| \leqslant \max _{i}\left|\left\langle x^{*}, k_{i} /(r-\varepsilon)\right\rangle\right|=\max _{i}\left|\left\langle x^{*}, h_{i}\right\rangle\right|
$$


that is, $x^{*} \in K\left(h_{1}, . ., h_{m}\right)$.

Conversely, if $\operatorname{dom} g_{v}^{*} \subseteq K$, then $\left(\operatorname{rec} g_{v}\right)^{0}=\overline{\text { cone }}^{w^{*}} \operatorname{dom} g_{v}^{*} \subseteq K$ and hence ${ }^{o} K \subseteq$ rec $g_{v}$. As $\left({ }^{\circ} K\right)^{\circ}=K$ is weak* locally compact, it follows from [10, Lemma 2.1] that $B(0, r) \subseteq{ }^{\circ} K-H$ for some $r>0$ and compact $H$. Hence (4.5) obtains for such $v$.

We have arrived at our first statement of a new sum theorem for slice convergence.

CoRollary 4.9. Let $\left\{f_{v}\right\}_{v \in W}$ and $\left\{g_{v}\right\}_{v \in W}$ be families of proper closed convex extended-real-valued functions on a Banach space $X$, which are slice convergent (as $v \rightarrow w)$ to $f_{w}$ and $g_{w}$ respectively. Assume also the following:

1. $f_{v}$ and $g_{v}$ are uniformly bounded below on $X$;

2. $\left\{g_{v}\right\}_{v \in W}$ satisfies condition (4.5);

3. $\overline{\text { cone }}\left(\operatorname{dom} f_{w}-\operatorname{dom} g_{w}\right)=X$.

Then $f_{v}+g_{v}$ is proper for all $v$ near $w$, and is slice convergent to $f_{w}+g_{w}$ as $v \rightarrow w$.

Proof: We prove that

$$
(\exists V \in \mathcal{N}(w))(\forall K \geqslant 1)\left(\bigcup_{v \in V} H_{K}(v) \text { is bounded }\right) .
$$

Clearly this will imply the second condition of Theorem 4.3. That it also implies the first, follows from Lemma 4.1 for each $v \in V$.

To verify (4.6), we suppose it false and derive a contradiction. If so, then there are nets $v_{\beta} \rightarrow w, K_{\beta} \in \mathbf{R}$, and $\left(x_{1_{\beta}}^{*}, x_{2_{\beta}}^{*}\right) \in H_{K_{\beta}}\left(v_{\beta}\right)$ with $\left\|x_{1_{\beta}}^{*}\right\|,\left\|x_{2_{\beta}}^{*}\right\| \rightarrow+\infty$, and $\lim _{\beta} K_{\beta} /\left\|x_{1_{\beta}}^{*}\right\|=0$.

To see this, note that (4.6) is false if and only if

$$
(\forall V \in \mathcal{N}(w))(\exists K \geqslant 1)(\forall \delta \geqslant 0)(\exists v \in V)\left(\exists\left(x_{1}^{*}, x_{2}^{*}\right) \in H_{K}(v)\right)\left(\left\|\left(x_{1}^{*}, x_{2}^{*}\right)\right\| \geqslant \delta\right),
$$

and from the freedom in $\delta \geqslant 0$ we may instead choose that $\left\|\left(x_{1}^{*}, x_{2}^{*}\right)\right\| \geqslant \delta+K^{2}$ in (4.7). Since $\mathcal{N}(w) \times(0,+\infty)$ is a directed set under the partial order:

$$
(V, \delta) \leqslant\left(V^{\prime}, \delta^{\prime}\right) \text { if and only if both } V^{\prime} \subseteq V \text { and } \delta^{\prime} \geqslant \delta
$$

(representing the limit as $V \downarrow\{w\}$ and $\delta \rightarrow+\infty)$, we have nets $v_{(V, \delta)} \rightarrow w$, $K_{(V, \delta)}:=K_{V} \geqslant 1,\left(x_{i_{(V, \delta)}^{*}}^{*}, x_{2_{(V, \delta)}}^{*}\right) \in H_{K_{V}}\left(v_{(V, \delta)}\right)$ with $\left\|\left(x_{i_{(V, \delta)}^{*}}^{*}, x_{2_{(V, \delta)}}^{*}\right)\right\| \geqslant \delta+K_{V}^{2}$. As $\left\|x_{1_{(V, \delta)}^{*}}^{*}+x_{2_{(V, \delta)}^{*}}^{*}\right\| \leqslant K_{V}$, then $\left\|x_{i_{(V, \delta)}^{*}}^{*}\right\| \geqslant \delta+K_{V}^{2}-K_{V}$, so $\lim _{(V, \delta)}\left\|x_{1_{(V, \delta)}^{*}}^{*}\right\|=+\infty$. Further, $K_{V} /\left\|x_{1_{(V, \delta)}}^{*}\right\| \leqslant K_{V} /\left(\delta+K_{V}^{2}-K_{V}\right) \rightarrow 0$ if $K_{(V, \delta)} \rightarrow+\infty$, and if $K_{(V, \delta)}$ does not converge to $+\infty$, so it has a bounded subnet, then along this subnet, $\underset{(V, \delta)}{\limsup } K_{V} /\left\|x_{1_{(\mathrm{V}, \delta)}}^{*}\right\|=0$, since $\left\|x_{1_{(V, \delta)}}^{*}\right\| \rightarrow+\infty$. In either case, this gives the desired construct.

Place $\bar{x}_{1_{\beta}}^{*}:=x_{1_{\beta}}^{*} /\left\|x_{1_{\beta}}^{*}\right\|$ and $\bar{x}_{2_{\beta}}^{*}:=x_{2_{\beta}}^{*} /\left\|x_{1_{\beta}}^{*}\right\|$. Then $\left\|\bar{x}_{1_{\beta}}^{*}+\bar{x}_{2_{\beta}}^{*}\right\| \leqslant K_{\beta} /\left\|x_{1_{\beta}}^{*}\right\| \rightarrow 0$. Clearly the $\bar{x}_{1_{\beta}}^{*}$ and $\bar{x}_{2_{\beta}}^{*}$ are bounded. Passing to a subnet, we may assume that $\bar{x}_{1_{\beta}}^{*} \stackrel{w^{*}}{\rightarrow} u^{*}$ 
and $\bar{x}_{2_{\beta}}^{*} \stackrel{w^{*}}{\rightarrow} v^{*}$ for some $u^{*}$ and $v^{*}$ which must then be in the unit ball and satisfy $u^{*}+v^{*}=0$.

Let $\lambda \geqslant 0$. We first assert that

$$
f_{w}^{*}\left(\lambda u^{*}\right) \leqslant \liminf _{\beta} f_{v_{\beta}}^{*}\left(\lambda \bar{x}_{1_{\beta}}^{*}\right)
$$

Indeed, for any $\mu \in \mathbf{R}$ such that $+\infty>\mu>\liminf _{\beta} f_{v_{\beta}}^{*}\left(\lambda \bar{x}_{1_{\beta}}^{*}\right),\left\{\beta \mid f_{v_{\beta}}^{*}\left(\lambda \bar{x}_{1_{\beta}}^{*}\right)<\mu\right\}$ is cofinal and so indexes a subnet. Therefore $\left(\lambda u^{*}, \mu\right) \in b w^{*}-\limsup$ epi $f_{v}^{*}$, and hence is in epi $f_{w}^{*}$ by Corollary 3.6. The claim then follows from the arbitrariness of $\mu$.

Similarly, $g_{w}^{*}\left(-\lambda u^{*}\right)=g_{w}^{*}\left(\lambda v^{*}\right) \leqslant \liminf _{\beta} g_{v_{\beta}}^{*}\left(\lambda \bar{x}_{2_{\beta}}^{*}\right)$. By convexity, since $0 \in \operatorname{dom} f_{v_{\beta}}^{*} \cap$ dom $g_{v_{\beta}}^{*}$ eventually in $\beta$,

$$
\begin{aligned}
f_{v_{\beta}}^{*}\left(\lambda \bar{x}_{1_{\beta}}^{*}\right)+g_{v_{\beta}}^{*}\left(\lambda \bar{x}_{2_{\beta}}^{*}\right) & \leqslant \frac{\lambda}{\left\|x_{1_{\beta}}^{*}\right\|}\left(f_{v_{\beta}}^{*}\left(x_{1_{\beta}}^{*}\right)+g_{v_{\beta}}^{*}\left(x_{2_{\beta}}^{*}\right)\right)+\left(1-\frac{\lambda}{\left\|x_{1_{\beta}}^{*}\right\|}\right)\left(f_{v_{\beta}}^{*}(0)+g_{v_{\beta}}^{*}(0)\right) \\
& \leqslant \lambda K_{\beta} /\left\|x_{1_{\beta}}^{*}\right\|+\sup _{v \in V}\left(f_{v}^{*}(0)+g_{v}^{*}(0)\right) \mid:=\lambda K_{\beta} /\left\|x_{1_{\beta}}^{*}\right\|+\xi
\end{aligned}
$$

for all $\beta$ such that also $\lambda /\left\|x_{1_{\beta}}^{*}\right\| \leqslant 1$, and where the latter supremum is taken over a neighbourhood of $w$, as per assumption (1). Then

$$
\begin{aligned}
f_{w}^{*}\left(\lambda u^{*}\right)+g_{w}^{*}\left(-\lambda u^{*}\right) & \leqslant \liminf _{\beta} f_{v_{\beta}}^{*}\left(\lambda \bar{x}_{1_{\beta}}^{*}\right)+\liminf _{\beta} g_{v_{\beta}}^{*}\left(\lambda \bar{x}_{2_{\beta}}^{*}\right) \\
& \leqslant \liminf _{\beta}\left(f_{v_{\beta}}^{*}\left(\lambda \bar{x}_{1_{\beta}}^{*}\right)+g_{v_{\beta}}^{*}\left(\lambda \bar{x}_{2_{\beta}}^{*}\right)\right) \\
& \leqslant \liminf _{\beta} \lambda K_{\beta} /\left\|x_{1_{\beta}}^{*}\right\|+\xi=\xi .
\end{aligned}
$$

Since $\lambda \geqslant 0$ is arbitrary, and $\xi$ is independent of $\lambda$,

$$
\begin{aligned}
u^{*} & \in \operatorname{rec}\left[f_{w}^{*}+g_{w}^{*}(-\cdot)\right] \\
& =\left(\operatorname{cone}\left(\operatorname{dom} f_{w}-\operatorname{dom} g_{w}\right)\right)^{\circ}=\{0\} \text { by Lemma } 4.7,(3) \text { and }(1)
\end{aligned}
$$

Since $\bar{x}_{2_{\beta}}^{*} \in \operatorname{dom} g_{v_{\beta}}^{*} \subseteq K\left(h_{1}, \ldots, h_{m}\right)$ for all $\beta$, the convergence $\bar{x}_{2_{\beta}}^{*} \stackrel{w^{*}}{\rightarrow} v^{*}=-u^{*}=0$ now implies $\left\|\bar{x}_{2_{\beta}}^{*}\right\| \rightarrow 0$, and hence that $1=\left\|\bar{x}_{1_{\beta}}^{*}\right\| \rightarrow 0$, a nonsense.

This sum property holds for the upper slice topology under much weaker hypotheses.

LEMMA 4.10. Suppose that $X$ is a normed space, $f_{v} \stackrel{\tau_{s}^{+}}{\rightarrow} f_{w}$ and $g_{v} \stackrel{\tau_{s}^{+}}{\rightarrow} g_{w}$ in $\Gamma(X)$, with dom $f_{v} \cap \operatorname{dom} g_{v}$ nonempty for each $v$ near $w$. Assume also that $\overline{f_{w}^{*} \square g_{w}^{*}}{ }^{*}=\overline{f_{w}^{*} \square g_{w}^{*}}$. Then $f_{v}+g_{v} \stackrel{\tau_{s}^{+}}{\rightarrow} f_{w}+g_{w}$.

PROOF: Note that $\left(f_{w}+g_{w}\right)^{*}=\overline{f_{w}^{*} \square g_{w}^{*} w^{*}}=\overline{f_{w}^{*} \square g_{w}^{*}}$. Let $\left(y^{*}, \alpha\right) \in \operatorname{epi}_{s}\left(f_{w}+g_{w}\right)^{*}$. Let $\gamma$ satisfy $\left(\overline{f_{w}^{*} \square g_{w}^{*}}\right)\left(y^{*}\right)<\gamma<\alpha$ and $\varepsilon$ be such that $\gamma+\varepsilon \leqslant \alpha$. Let $\left\{x_{\beta}\right\} \subseteq B(0, M)$ be a bounded net, and $v_{\beta} \rightarrow w$. There is $\bar{y}^{*}$ such that $\left\|\bar{y}^{*}-y^{*}\right\| \leqslant \varepsilon / M$ with $\left(f_{w}^{*} \square g_{w}^{*}\right)\left(\bar{y}^{*}\right)<$ 
$\gamma$. Then $\left(\bar{y}^{*}, \gamma\right) \in \operatorname{epi}_{s} f_{w}^{*} \square g_{w}^{*}$ has the decomposition $\left(y_{1}^{*}, \gamma_{1}\right)+\left(y_{2}^{*}, \gamma_{2}\right) \in \operatorname{epi}_{s} f_{w}^{*}+\operatorname{epi}_{s} g_{w}^{*}$. Hence we obtain

$$
\begin{aligned}
\left(f_{v_{\beta}}+g_{v_{\beta}}\right)\left(x_{\beta}\right) & >\left\langle y_{1}^{*}, x_{\beta}\right\rangle-\gamma_{1}+\left\langle y_{2}^{*}, x_{\beta}\right\rangle-\gamma_{2} \\
& =\left\langle\bar{y}^{*}, x_{\beta}\right\rangle-\gamma \geqslant\left\langle y^{*}, x_{\beta}\right\rangle-M\left\|\bar{y}^{*}-y^{*}\right\|-\gamma \\
& \geqslant\left\langle y^{*}, x_{\beta}\right\rangle-(\varepsilon+\gamma)>\left\langle y^{*}, x_{\beta}\right\rangle-\alpha
\end{aligned}
$$

eventually in $\beta$.

Since upper slice convergence roughly corresponds to (strong) lower-Kuratowski convergence of the conjugates (Theorem 3.5), the above lemma may be viewed as a result on epi-upper-semicontinuity of inf-convolutions (see [22, Lemma 4.1]).

We now state our main sum theorem for slice convergence.

THEOREM 4.11. Let $\left\{f_{v}\right\}_{v \in W}$ and $\left\{g_{v}\right\}_{v \in W}$ be families of proper closed convex extended-real-valued functions on a Banach space $X$, which are slice convergent (as $v \rightarrow w)$ to $f_{w}$ and $g_{w}$ respectively. Assume also the following:

1. $\left\{g_{v}\right\}_{v \in W}$ satisfies condition (4.5);

2. $\overline{\text { cone }}\left(\operatorname{dom} f_{w}-\operatorname{dom} g_{w}\right)=X$;

3. $\overline{f_{w}^{*} \square g_{w}^{*}} \omega^{*}=\overline{f_{w}^{*} \square g_{w}^{*}}$.

Then $\left\{f_{v}+g_{v}\right\}_{v \in W}$ is slice convergent to $f_{w}+g_{w}$ as $v \rightarrow w$.

Proof: Let $M \in \mathbf{R}$. Then $f_{v} \vee M:=\max \left\{f_{v}, M\right\}$ and $g_{v} \vee M$ are uniformly bounded below in $x \in X$ and $v \in W$. Also, $\operatorname{dom} f_{v} \vee M=\operatorname{dom} f_{v}$ and similarly for $g_{v}$. Since $\left\{g_{v} \vee M \leqslant \lambda\right\}=\left\{g_{v} \leqslant \lambda\right\}$ for all $\lambda \geqslant M$, then $\operatorname{rec} g_{v} \vee M=\operatorname{rec} g_{v}$. Further, we have the slice convergence $f_{v} \vee M \rightarrow f_{w} \vee M$ and $g_{v} \vee M \rightarrow g_{w} \vee M$, by Corollary 4.5 (considered as a result on the slice convergence of intersections of sets, applied to epi $f_{v} \vee M=$ epi $\left.f_{v} \cap(X \times[M,+\infty))\right)$. We may now apply Corollary 4.9 to infer that $f_{v} \vee M+g_{v} \vee M$ is proper for $v$ near $w$, and slice-converges to $f_{w} \vee M+g_{w} \vee M$. In particular, $\operatorname{dom} f_{v}$ intersects dom $g_{v}$, and

$$
f_{w} \vee M+g_{w} \vee M \geqslant \underset{v \rightarrow w}{\operatorname{e-l}}\left(f_{v} \vee M+g_{v} \vee M\right) \geqslant \underset{v \rightarrow w}{\operatorname{e}-\operatorname{ls}}\left(f_{v}+g_{v}\right)
$$

and since $M$ is arbitrary,

$$
f_{w}+g_{w} \geqslant \underset{v \rightarrow w}{\mathrm{e}-l \mathrm{ls}}\left(f_{v}+g_{v}\right)
$$

The upper slice convergence follows from Lemma 4.10.

REMARK 4.3. Note that (3) is automatically satisfied in reflexive $X$. If $X$ is not reflexive, this condition can be taken care of as follows: If (2) is strengthened to

$$
\text { cone }\left(\operatorname{dom} f_{w}-\operatorname{dom} g_{w}\right)=X,
$$


then $f_{w}^{*} \square g_{w}^{*} \in \Gamma^{*}\left(X^{*}\right)$ by [3]; alternately, if instead, $f_{w}$ and $g_{w}$ are assumed bounded below on $X$, we may apply the argument of Corollary 4.9 with $W=\{w\}$ to deduce that $H_{K}(w)$ is bounded for each $K$, implying by Lemma 4.1 that $f_{w}^{*} \square g_{w}^{*}$ is in $\Gamma^{*}\left(X^{*}\right)$.

Another option is to assume some (weak) compactness condition on the limit functions, and to apply the following

LEMMA 4.12. Let $f$ and $g$ be in $\Gamma(X)$, with intersecting domains, and such that for each $K \geqslant 0$, dom $f \cap B(0, K)$ is relatively weakly compact. Then

$$
\overline{f^{*} \square g^{*}} \omega^{*}=\overline{f^{*} \square g^{*}} \text {. }
$$

PRoOF: The assumption on the domain implies that for each $K, \overline{\operatorname{dom} f} \cap \bar{B}(0, K)$ is weakly compact. Letting $J: X \rightarrow X^{* *}$ denote the canonical embedding, $J(\overline{\operatorname{dom} f}) \cap$ $\bar{B}^{* *}(0, K)=J(\overline{\operatorname{dom} f} \cap \bar{B}(0, K))$ is then $\sigma\left(X^{* *}, X^{*}\right)$-closed in $X^{* *}$. Indeed, if $J\left(x_{\beta}\right) \stackrel{w^{*}}{\rightarrow} x^{* *}$ with $x_{\beta} \in \overline{\operatorname{dom} f} \cap \bar{B}(0, K)$, then by weak compactness $x_{\beta} \stackrel{w}{\rightarrow} x \in \overline{\operatorname{dom} f} \cap \bar{B}(0, K)$ (along a subnet), or equivalently $J\left(x_{\beta}\right) \stackrel{w^{*}}{\rightarrow} J(x)$, so $x^{* *}=J(x) \in J(\overline{\operatorname{dom} f} \cap \bar{B}(0, K))$.

Hence $J(\overline{\operatorname{dom} f})=\overline{J(\operatorname{dom} f)}$ is $\sigma\left(X^{* *}, X^{*}\right)$-closed in $X^{* *}$ by the Krein-Šmulian Theorem, with the consequence that the $\sigma\left(X^{* *}, X^{*}\right)$-closure of $J(\operatorname{dom} f)$ is in $J(X)$. Place $\psi:=f^{*} \square g^{*}$. Then since $\psi^{*}=f+g \in \Gamma(X)$, it follows that $\Gamma^{*}\left(X^{*}\right) \ni \psi^{* *}=\bar{\psi}^{w^{*}}$ (the $\sigma\left(X^{*}, X\right)$-1.s.c. hull of $\psi$ ), which implies that $\psi$ is also proper. Hence also the $\sigma\left(X^{*}, X^{* *}\right)$-l.s.c. hull $\vec{\psi}^{\omega}$ is proper, since $\vec{\psi}^{\omega^{*}} \leqslant \bar{\psi}^{w} \leqslant \psi$.

For emphasis, we distinguish the conjugation operator relative to the standard pairing of $\left(X^{*}, \sigma\left(X^{*}, X^{* *}\right)\right)$ with $\left(X^{* *}, \sigma\left(X^{* *}, X^{*}\right)\right)$ by writing it as ${ }^{*}$, with * reserved for the operation relative to the pairing of $\left(X, \sigma\left(X, X^{*}\right)\right)$ with $\left(X^{*}, \sigma\left(X^{*}, X\right)\right)$. Then for any function $\varphi$ on $X$,

$$
\widetilde{\varphi}^{\bar{*}}=\varphi^{*}
$$

where $\tilde{\varphi}: X^{* *} \rightarrow \overline{\mathbf{R}}$ is defined by

$$
\widetilde{\varphi}= \begin{cases}\varphi\left(J^{-1}(\cdot)\right) & \text { on } J(X) \\ +\infty & \text { on } X^{* *} \backslash J(X) .\end{cases}
$$

Then

$$
h:=\psi^{\bar{*}}=\left(f^{*} \square g^{*}\right)^{\bar{*}}=\left(\widetilde{f}^{\bar{*}} \square \widetilde{g}^{\bar{*}}\right)^{\bar{*}}=\widetilde{f}^{\bar{*}}+\widetilde{g}^{\overline{* *}}=\widetilde{\vec{f}}^{*}+\widetilde{\widetilde{g}}^{*},
$$

where the latter summands are $\sigma\left(X^{* *}, X^{*}\right)$-l.s.c. hulls, and since their domains intersect, $h$ is proper, with

$$
\operatorname{dom} h \subseteq \operatorname{dom} \overrightarrow{\widetilde{f}}^{\omega^{*}} \subseteq \overline{\operatorname{dom}} \vec{f}^{\omega^{*}}=\overline{J(\operatorname{dom} f)}^{\sigma\left(X^{*}, X^{*}\right)} \subseteq J(X)
$$

so $h=\tilde{h}_{0}$ for some proper $h_{0}: X \rightarrow \overline{\mathrm{R}}$. Thus $\bar{\psi}^{\omega}=\psi^{\bar{*}}=h^{\bar{*}}=h_{0}^{*}$, the latter being $\sigma\left(X^{*}, X\right)$-closed, and so $\bar{\psi}^{\omega^{*}}=\bar{\psi}^{\omega}$, which coincides with the strong closure $\bar{\psi}$ by convexity.

The above Theorem takes a neat form for the slice convergence of subspaces. 
Corollary 4.13. Let $M_{v}$ and $N_{v}(v \in W)$ be closed subspaces of the Banach space $X$, slice convergent (as $v \rightarrow w$ ) to $M_{w}$ and $N_{w}$ respectively, such that also $\overline{M_{w}+N_{w}}=X$, and for some finite-dimensional subspace $F \subseteq X^{*}$ and neighbourhood $V$ of $w, \bigcup_{v \in V} N_{v}^{\perp} \subseteq F$. Then $M_{v} \cap N_{v}$ slice converges to $M_{w} \cap N_{w}$.

Proof: The condition on the $N_{v}^{\perp}$ is equivalent to assumption (1) of the Theorem. Indeed, if (1) holds for $N_{v}$, then by Lemma 4.8, $N_{v}^{\perp} \subseteq K$ (for $v$ near $w$ ) for some weakly* locally compact cone $K$, and hence $N_{v}^{\perp} \subseteq K \cap-K:=F^{\prime}$, a weakly* locally compact subspace, which must therefore be of finite dimension. The second part of the proof of Lemma 4.8 yields the reverse argument, by the weak ${ }^{*}$ local compactness of finitedimensional subspaces.

We note that in general, the result does not hold if the uniform finite-codimensionality condition (1) is omitted. For example, (see [7, Exercise 8.1.6]) let $X=l^{2}, M_{n}:=\{x \in$ $\left.l^{2} \mid x_{n+1}=0\right\}, N_{n}:=\left\{x \in l^{2} \mid x_{n+1}=x_{1} / n\right\}$. Then $M_{n}$ and $N_{n}$ are closed subspaces slice converging to $M=N=X$. However, $M_{n} \cap N_{n}=\left\{x \in l^{2} \mid x_{1}=0, x_{n+1}=0\right\}$, which slice converges to $\left\{x \in l^{2} \mid x_{1}=0\right\} \neq X=M \cap N$. Since $M_{n}^{\perp}=$ span $e_{n+1}$ and $N_{n}^{\perp}=\operatorname{span}\left(e_{1}-n e_{n+1}\right)$ for each $n$ (where $e_{n}$ denote the standard basis elements in $l^{2}$ ), the uniform containment of $M_{n}^{\perp}$ or $N_{n}^{\perp}$ in any fixed finite-dimensional subspace is not possible.

This example also shows that any proposal to weaken the assumption in Corollary 4.4 to, say,

$$
B(0, \delta) \subseteq\left\{f_{v} \leqslant \mu\right\}-\left\{g_{v} \leqslant \mu\right\}
$$

for $v$ near $w$, will in general, fail to yield slice convergence for the sum $f_{v}+g_{v}$, since for all $n, M_{n}+N_{n}=X$, so the indicator functions certainly satisfy this relation.

\section{An Application to $l^{1}$ Control Problems}

We end with a brief application, which resolves an issue in the theory of $l^{1}$-optimal control. All the system-theoretic concepts to be discussed can be found in detail in: [13] for general systems theory; [23] for general control theory for linear systems; and [12] for $l^{1}$-optimal control.

We shall restrict ourselves to single-input/single-output linear time-invariant (LTI) discrete-time systems.

Such systems always take the form of a convolution operator $h *$ for some real sequence $h=\left\{h_{i}\right\}_{i=0}^{\infty}$ (called the pulse response, since $h=h * \delta$ where $\delta:=(1,0,0, \ldots)$ is the unit pulse), with the operator acting on the space of all real sequences. It is known that this operator is BIBO (bounded-input/bounded-output) stable (that is, $h * e \in l^{\infty}$ $\left.\forall e \in l^{\infty}\right)$ if and only if $h \in l^{1}[13]$.

Let $P$ be a LTI system (the 'plant' to be controlled). A system $K$ is said to (BIBO-)stabilise $P$ if the closed-loop system depicted in Figure 1 is BIBO-stable in 
the sense that for any input $w \in l^{\infty}$ and any bounded disturbances applied additively at any point in the loop, all resulting signals within the loop are also bounded [13], [23].

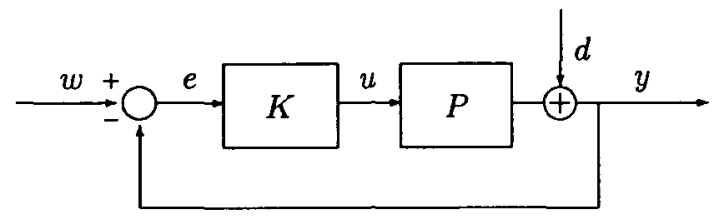

Figure 1. A closed-loop control system

The stated control problem amounts to finding, among all controllers $K$ stabilising $P$, that which performs best according to some chosen criterion. If we restrict $P$ and $K$ to have rational $z$-transforms $\widehat{P}, \widehat{K}$ (where $\widehat{P}(z):=\sum_{i=0}^{\infty} P_{i} z^{i}$ for $z \in \mathrm{C}$ inside the radius of convergence of the series) then by the use of the YJBK parametrisation [23] for the set of all stabilising controllers $K$, the corresponding set of error signals $e$ has the structure of an affine set. It should be noted that there is a one-to-one correspondence between an error $e$ and the associated $K$, with a simple algebraic equation connecting them. Hence the control problem can be restated as a minimisation over the affine space of error signals, and hence is in a form nearer to that which can be treated by the traditional techniques of optimisation theory.

If further, we require $e \in l^{1}$ (this represents, if $w \notin l^{1}$, a "tracking" requirement for the output, and is superfluous if $w \in l^{1}$ ), then the set of prospective candidates for the minimisation can be expressed as the subset of members with rational $z$-transform, of $\left\{e \in l^{1} \mid A e=b\right\}$, where $b \in \mathbf{R}^{n}$ and $A: l^{1} \rightarrow \mathbf{R}^{n}$ is bounded linear [12]. In the general "multiblock" case, (which we do not consider here), $A$ would map into some infinitedimensional space $Z$, with $b \in Z$, where $A$ usually has closed range-for more on this, see $[12]$.

If we also want to apply time-domain constraints on $e$, by requiring that $e \in C:=$ $\left\{e \in l^{1} \mid B_{i} \leqslant e_{i} \leqslant A_{i}\right.$ for all $\left.i\right\}$, this leads to the minimisation

$$
\inf _{e \in C \cap M} f(e)
$$

for some performance measure $f$, say, the $l^{\mathbf{1}}$-norm. This is the abstract form of what is computed in $l^{1}$-optimal control (see $[12, \mathbf{1 5}]$ ). However, since the YJBK parametrisation applies only to $K$ with rational $z$-transform (which then corresponds to rational $e$ ), we observe that generally, (5.1) is merely a lower bound for the best performance by 'physically realisable' controllers (that is, those with rational transform). This leads to the important question of whether equality obtains, that is:

$$
\inf _{C \cap M} f=\inf \{f(e) \mid e \in C \cap M, \hat{e} \text { rational }\}
$$


We henceforth assume that $f$ is continuous on $l^{1}$. Let $X_{0}:=\left\{e \in l^{1} \mid \hat{e}\right.$ is rational $\}$ (a subspace of $l^{1}$ ). Since it can be shown without much difficulty that $M \cap X_{0}$ is dense in $M$, it follows by an elementary convexity argument that if $M \cap \operatorname{int} C$ is nonempty, then $\overline{C \cap M \cap X_{0}}=C \cap M$, so the required equality follows in this case. If $C$ lacks interior, things are a bit less obvious.

From use of a duality theorem of Borwein and Lewis [11] in both $X$ and $X_{0}$, it can be shown that (5.2) obtains if qri $(C) \cap M \cap X_{0} \neq \emptyset$. By use of our Corollary 4.4, we can find a complementary sufficient condition.

Corollary 5.1. If $\overline{\text { cone }}(C-M)=l^{1}$ then (5.2) follows.

Proof: Place $f_{n}:=\delta_{C_{n}}, C_{n}:=\left\{e \in C \mid e_{i}=0\right.$ for all $\left.i \geqslant n\right\}, g_{n}:=g:=\delta_{M}$. Then $f_{n}$ slice converges to $f$ and trivially $g_{n}$ converges. Since rec $M=M$ and $M$ has finite codimension, so $g_{n}$ satisfies (4.5), we may apply Corollary 4.9 to infer the slice convergence $C_{n} \cap M \rightarrow C \cap M$. In particular, $C \cap M \cap X_{0}$ is dense in $C \cap M$ since $C_{n}$ consists of elements of finite length, which must have rational $z$-transform.

\section{REFERENCES}

[1] H. Attouch, Variational convergence for functions and operators, Applicable Mathematics Series (Pitman, London, 1984).

[2] H. Attouch and R.J.-B. Wets, 'Quantitative stability of variational systems: III. E-approximate solutions', Math. Programming 61 (1993), 197-214.

[3] H. Attouch and H. Brézis, 'Duality for the sum of convex functions in general Banach spaces', in Aspects of Mathematics and its Applications, (J. Barroso, Editor) (Elsevier Science. Publishers, 1986), pp. 125-133.

[4] J.-P. Aubin and H. Frankowska, Set-valued analysis, Systems and Control: Foundations and Applications 2 (Birkhäuser, Boston, MA, 1990).

[5] D. Azé and J.-P. Penot, 'Operations on convergent families of sets and functions', Optimization 21 (1990), 521-534.

[6] G. Beer, 'The slice topology: A viable alternative to Mosco convergence in non-reflexive spaces', Nonlinear Anal. 19 (1992), 271-290.

[7] G. Beer, Topologies on closed and closed convex sets, Mathematics and its Applications 268 (Kluwer Acad. Publ., Dordrecht, 1993).

[8] G. Beer and M. Borwein, 'Mosco convergence and reflexivity', Proc. Amer. Math. Soc. 109 (1990), 427-436.

[9] G. Beer and R. Lucchetti, 'The Epi-distance topology: Continuity and stability results with applications to convex optimization problems', Math. Oper. Res. 17 (1992), 715-726.

[10] J.M. Borwein, 'Epi-Lipschitz-like sets in Banach space: Theorems and examples', Nonlinear Anal. 11 (1987), 1207-1217.

[11] J.M. Borwein and A.S. Lewis, 'Partially-finite convex programming', Math. Programming 57 (1992), 15-83.

[12] M.A. Dahleh and I.J. Diaz-Bobillo, Control of uncertain systems: A linear programming approach (Prentice-Hall, New Jersey, 1995). 
[13] C.A. Desoer and M. Vidyasagar, Feedback systems: Input-output properties (Academic Press, New York, London, 1975).

[14] N. Dunford and J.T. Schwartz, Linear operators I, General theory, Wiley-Interscience (J. Wiley and Sons, New York, 1957).

[15] N. Elia and M. A. Dahleh, 'Controller design with multiple objectives', IEEE Trans. Automat. Control AC-42 (1997), 596-613.

[16] R.B. Holmes, Geometric functional analysis and its applications, Graduate Texts in Mathematics 24 (Springer-Verlag, Berlin, Heidelberg, New York, 1975).

[17] A. Jourani, 'Compactly Epi-Lipschitzian sets and A-subdifferentials in WT-spaces', $O p$ timization 34 (1995), 1-17.

[18] P.D. Loewen, 'Limits of Fréchet normals in nonsmooth analysis', in Optimization and Nonsmooth Analysis, (Ioffe, Marcus, Reich, Editors), Research Notes in Mathematics Series 244 (Longman Sci. and Tech., Harlow 1990), pp. 177-188.

[19] J.-P. Penot, 'Preservation of persistence and stability under intersection and operations', J. Optim. Theory Appl. 79 (1993), 525-561.

[20] R.T. Rockafellar and J.-B. Wets, 'Variational systems, an introduction', in Multifunctions and integrands, (G. Salinetti, Editor), Lecture Notes in Mathematics 1091 (Springer-Verlag, Berlin, Heidelberg, New York, 1984), pp. 1-54.

[21] R.T. Rockafellar, 'Level-sets and continuity of conjugate convex functions', Trans. Amer. Math. Soc. 123 (1966), 46-61.

[22] T. Strömberg, 'The operation of infimal convolution', Dissertationes Math. 352 (1996), 4-58.

[23] M. Vidyasagar, Control system synthesis (MIT Press, Cambridge, 1985).

[24] S.L. Zabell, 'Mosco convergence in locally convex spaces', J. Funct. Anal. 110 (1992), 226-246.

Department of Mathematics

Royal Melbourne University of Technology

Melbourne Vic 3001

Australia

e-mail: andy.eb@rmit.edu.au 